Research Article

\title{
Development and Validation of an Autophagy-Related Gene Signature for Predicting the Prognosis of Hepatocellular Carcinoma
}

\author{
Jianlin Zhang $\mathbb{D}^{1},{ }^{1}$ Min Zhang $\mathbb{D}^{2}$ Jin Huang $\mathbb{D}^{3}{ }^{3}$ Gaosong Zhang $\mathbb{D}^{4}$, Chong $\mathrm{Li}_{\mathbb{D}},{ }^{4}$ \\ Xingyu Wang $\mathbb{D}^{1}$, and Weihao Kong $\mathbb{D}^{1}$ \\ ${ }^{1}$ Department of Emergency Surgery, Department of Emergency Medicine, The First Affiliated Hospital of Anhui Medical University, \\ Hefei, Anhui, China \\ ${ }^{2}$ Department of Anesthesiology, Jiulongpo People's Hospital, Chongqing, China \\ ${ }^{3}$ Department of Pathology, The Second People's Hospital of Hefei, Hefei, Anhui, China \\ ${ }^{4}$ Department of Ultrasound, The First Affiliated Hospital of Anhui Medical University, Hefei, Anhui, China
}

Correspondence should be addressed to Xingyu Wang; wangxingyu@ahmu.edu.cn

and Weihao Kong; kongwh3@mail2.sysu.edu.cn

Received 24 June 2021; Accepted 15 October 2021; Published 28 October 2021

Academic Editor: Maciej Gagat

Copyright (c) 2021 Jianlin Zhang et al. This is an open access article distributed under the Creative Commons Attribution License, which permits unrestricted use, distribution, and reproduction in any medium, provided the original work is properly cited.

Purpose. Autophagy is a lysosomal degradation pathway that is essential for maintaining the homeostasis of the intracellular environment. Mounting evidence indicates that autophagy plays an essential role in the occurrence and development of hepatocellular cancer (HCC). This research is aimed at exploring the prognostic value of autophagy-related genes (ARGs) in HCC patients. Methods. The Wilcoxon test was used to identify differentially expressed ARGs in The Cancer Genome Atlas (TCGA) HCC cohort. Then, the TCGA cohort was randomly divided into training and testing groups. Cox and LASSO regression models were used to screen for autophagy-related genes that affect overall survival (OS) in the TCGA training group. Based on the coefficient of risk genes, we constructed an autophagy-related gene signature for predicting the prognosis of HCC patients. Finally, we validated the prognostic significance of autophagy-related gene signature using the TCGA testing group and three external datasets. Results. ATG10, BIRC5, GAPDH, and TMEM74 are risk genes for OS. According to the optimal cutoff value of risk score in each HCC dataset, HCC patients can divide into high- and low-risk groups. ARG risk score can significantly distinguish HCC patients with different survival outcomes. Meanwhile, the ARG risk score is independently correlated with OS in multiple HCC cohorts. Conclusions. The autophagy-related risk score can effectively screen high-risk HCC patients and provide guidance for clinical prevention and treatment of HCC.

\section{Introduction}

Hepatocellular carcinoma (HCC) is the third leading cause of cancer-related death in China and the fourth leading cause of cancer-related death in the world. Despite significant advances in the diagnosis and treatment of HCC in recent years, the prognosis of hepatocellular carcinoma is still poor, owing to its high invasiveness $[1,2]$. Therefore, it is essential to explore the molecular mechanism of the occurrence and development of liver cancer, as it can lead to a new treatment strategy for the prevention and treatment of hepatocellular carcinoma.

Autophagy refers to the process of using lysosomes to degrade self-damaged organelles and macromolecules under the regulation of autophagy-related genes, thereby maintaining the needs of the cells themselves and the renewal of organelles. Changes in the level of autophagy are associated with a variety of human diseases, such as cancer, autoimmune diseases, and central nervous system diseases [3-6]. Several studies have shown that autophagy can inhibit the 
TABLE 1: Clinicopathological characteristics of the TCGA training group, the TCGA testing group, and the entire TCGA dataset.

\begin{tabular}{lccc}
\hline Variables & Entire dataset & Training group & Testing group \\
$n=367$ & $n=183$ & $P$ value \\
\hline Age (years) & $61.0(52.0-69.0)$ & $61.0(52.0-68.0)$ & $62.0(51.0-69.8)$ \\
Gender, male/female & $248 / 119$ & $125 / 58$ & $123 / 61$ \\
AJCC stage, I/II/III/IV/NA & $171 / 85 / 83 / 4 / 24$ & $86 / 51 / 37 / 1 / 8$ & 0.487 \\
Histologic grade, G4/G3/G2/G1/NA & $55 / 176 / 119 / 12 / 5$ & $25 / 89 / 61 / 5 / 3$ & 0.765 \\
Survival status, alive/dead & $237 / 130$ & $113 / 70$ & 0.090 \\
Survival time (months) & $19.7(11.3-36.3)$ & $18.9(11.4-34.5)$ & 0.897 \\
\hline
\end{tabular}

Notes: AJCC: American Joint Committee on Cancer; TCGA: The Cancer Genome Atlas.

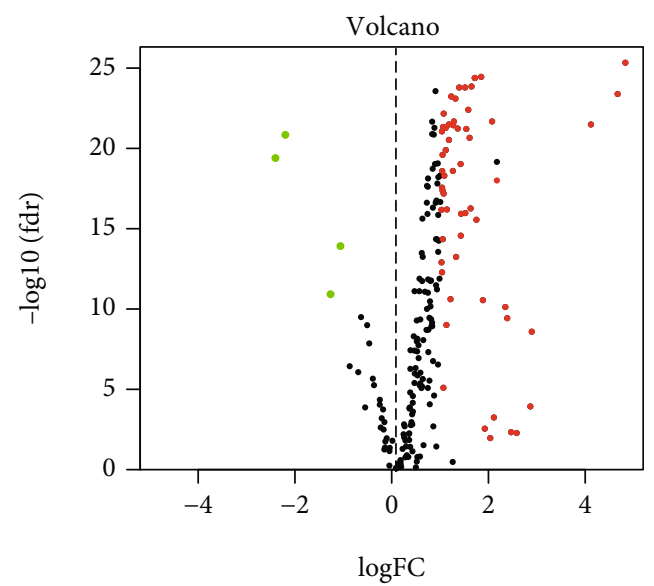

(a)

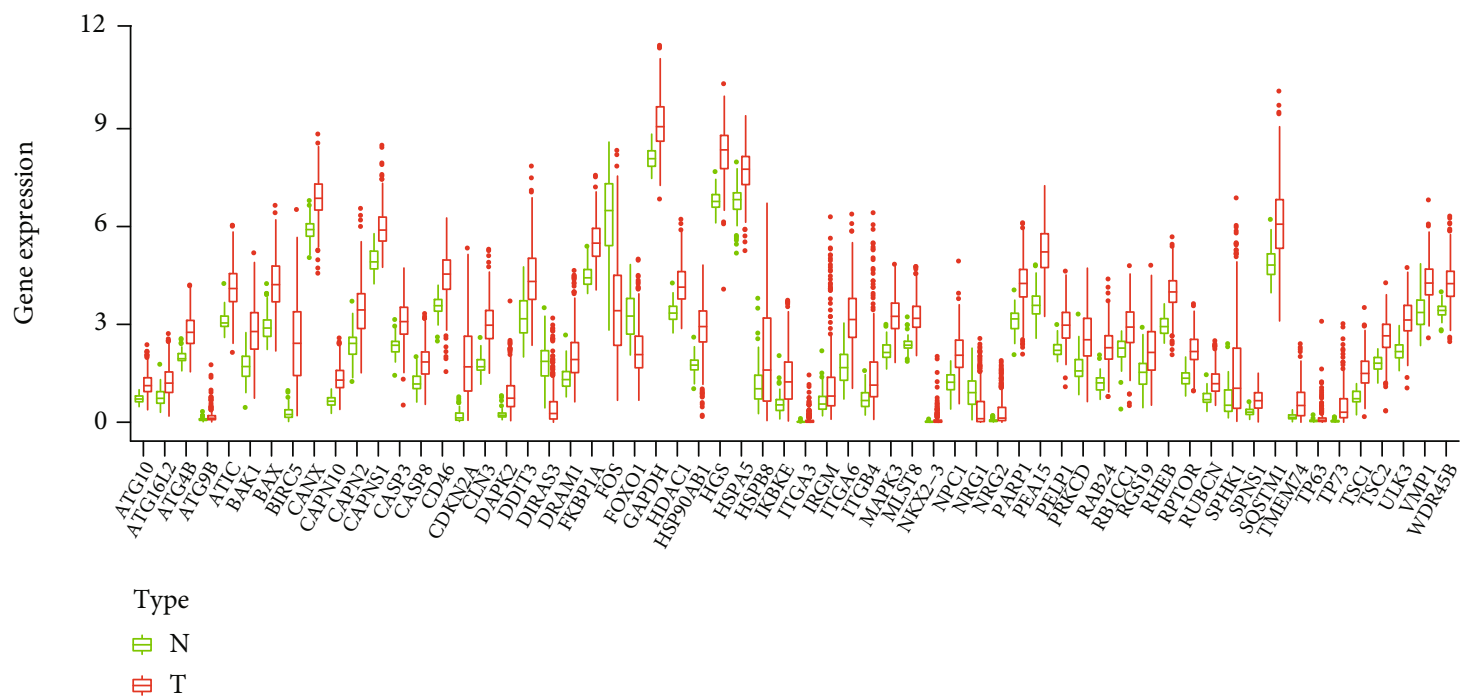

(b)

FIGURE 1: Differentially expressed analysis of autophagy-correlated genes in the TCGA HCC cohort. (a) Volcano plot of the autophagyrelated genes. Notes: TCGA: The Cancer Genome Atlas; HCC: hepatocellular carcinoma; red: upregulated genes; green: downregulated genes; black: nondifferentially expressed genes. (b) Expression of 62 differentially expressed autophagy-related genes in HCC patients and normal controls. Notes: HCC: hepatocellular carcinoma; red represents tumor samples, and the green represents normal controls.

growth and invasion of tumor cells in the early stages of many tumors. However, in the advanced tumor stages, autophagy can promote the rapid growth of tumor cells by degrading aging and damaged organelles and macromole- cules, thereby promoting the malignant transformation of tumor cells $[4,6]$. The change in autophagy level is closely related to the development of liver cancer. The main types of autophagy in the progression of liver cancer are mainly 


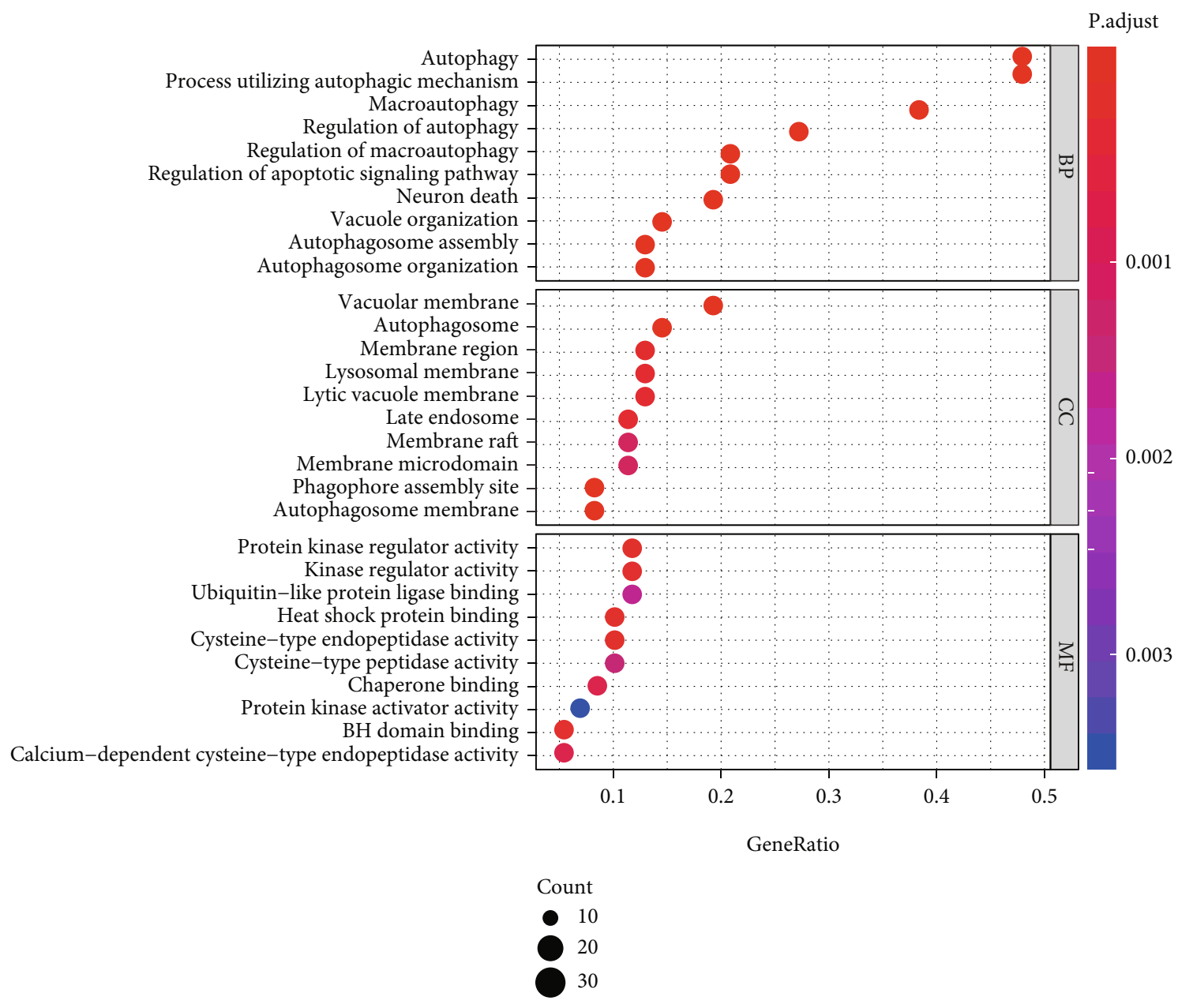

(a)

Figure 2: Continued. 


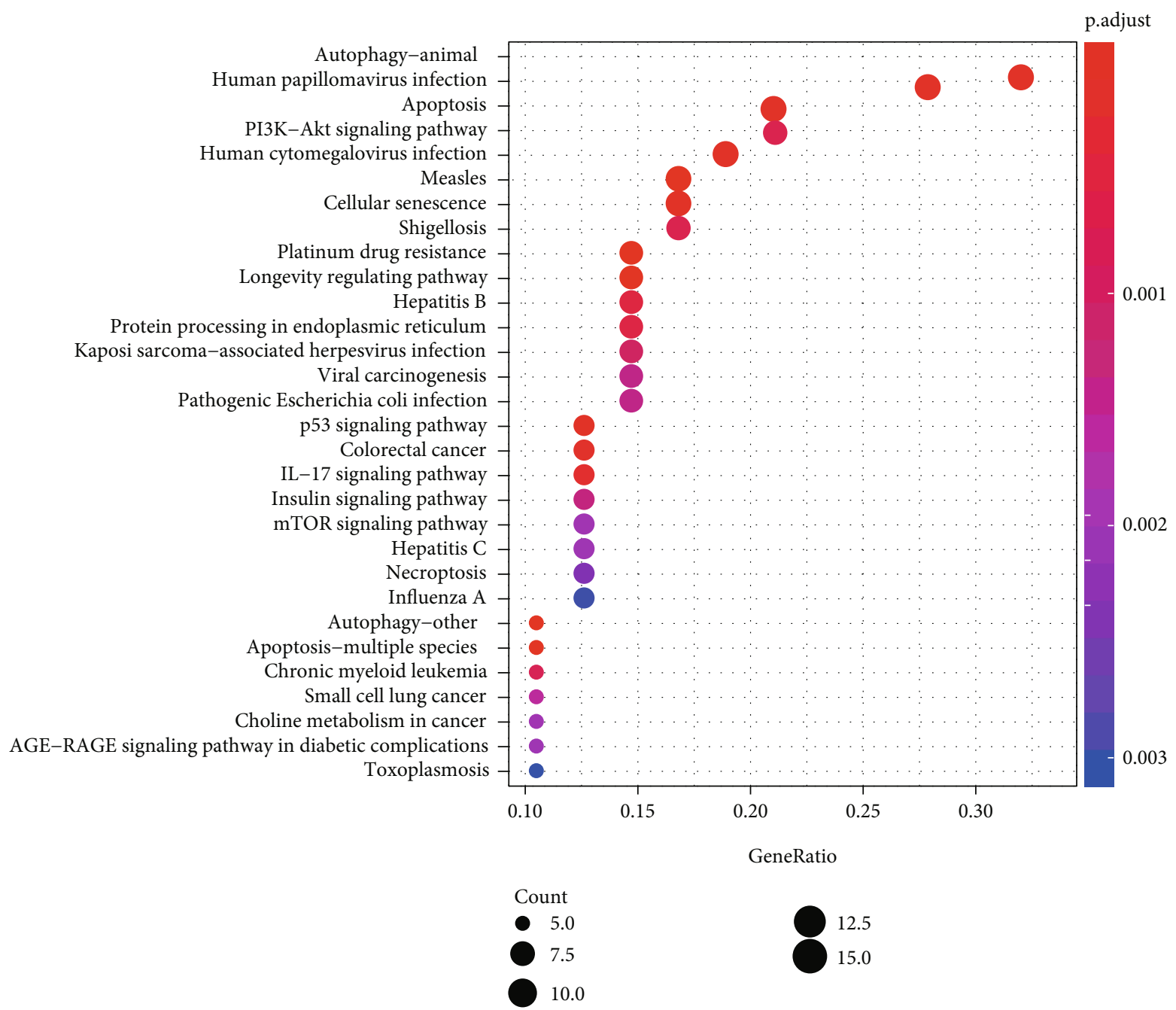

(b)

Figure 2: Gene enrichment analysis of GO (a) and KEGG (b). Notes: GO: Gene Ontology; KEGG: Kyoto Encyclopedia of Genes and Genomes.

molecular chaperone-mediated autophagy (CMA) and macroautophagy [7]. On the one hand, mice with weakened CMA can increase the vulnerability to oxidative stress, worsen liver function, and accelerate metabolic abnormalities, thereby promoting the occurrence of hepatic adenoma [8]. When it is progressing to a malignant tumor, the tumor cells show a significant increase in CMA activity in order to maintain the metabolic shift of cancerous cells [9]. On the other hand, macroautophagy plays an antitumor role in the progression of liver cancer. The reduction in autophagy level is associated with malignant transformation and poor prognosis of liver cancer. In liver cancer, a decrease in the level of autophagy can cause the appearance of the autophagy protein p62, and p62 can promote the release of nuclear factor erythroid 2-related factor 2 (Nrf2), which in turn encourages the progression of liver fibrosis and liver cancer $[10,11]$. Therefore, autophagy as a target for antitumor may have important clinical significance in the future. Since autophagy plays an essential role in the occurrence and development of cancer, it is of great clinical importance to find autophagy-related tumor biomarkers.

With the popularization of high-throughput sequencing technology in recent years, it is feasible to explore the relationship between autophagy-related genes (ARGs) and the prognosis of patients with hepatocellular carcinoma. Therefore, in our study, we systematically analyzed the differentially expressed ARGs in HCC by using the TCGA database and selected differentially expressed ARGs significantly associated with OS in the TCGA training group. Based on the cutoff value of risk score in the TCGA training group, HCC patients could be divided into high- and lowrisk groups. Finally, we explored the prognostic role of the ARG risk score in the TCGA training group, TCGA testing group, and three external datasets.

\section{Material and Methods}

2.1. Data Acquisition. The Human Autophagy Database (http://autophagy.lu/clustering/index.html) is a specialized 


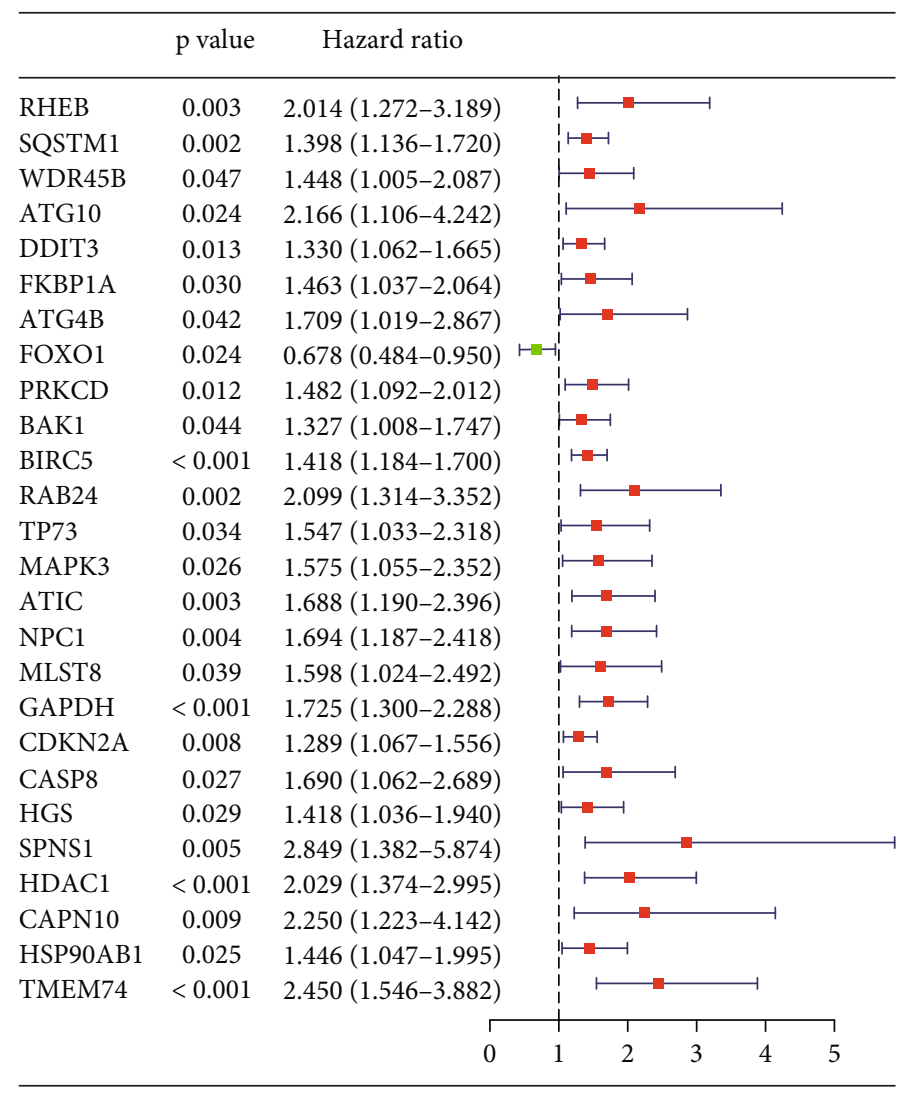

(a)

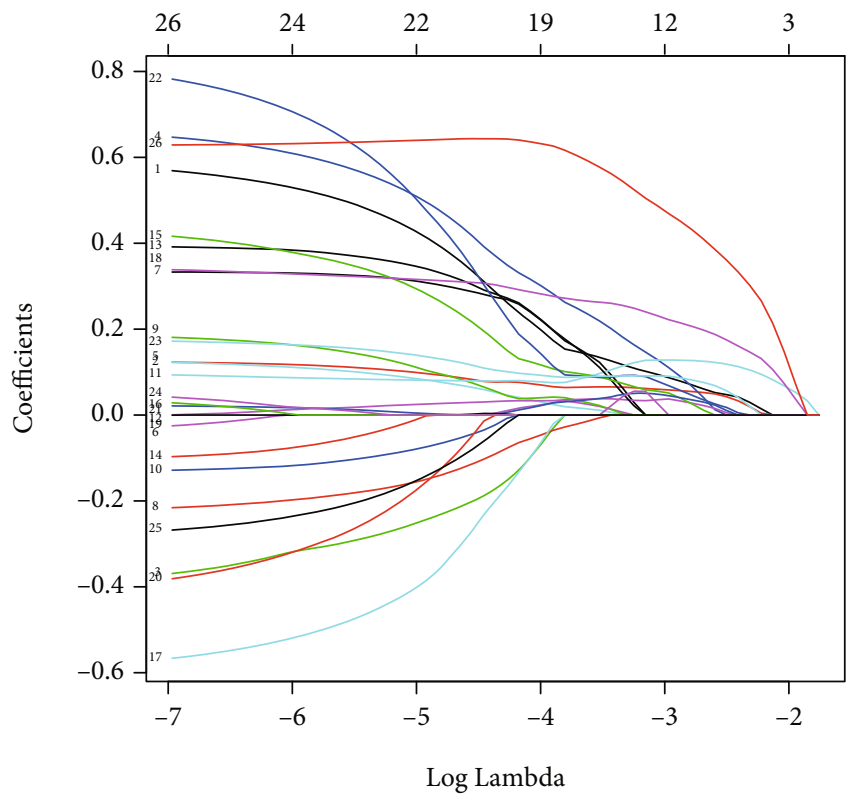

(b)

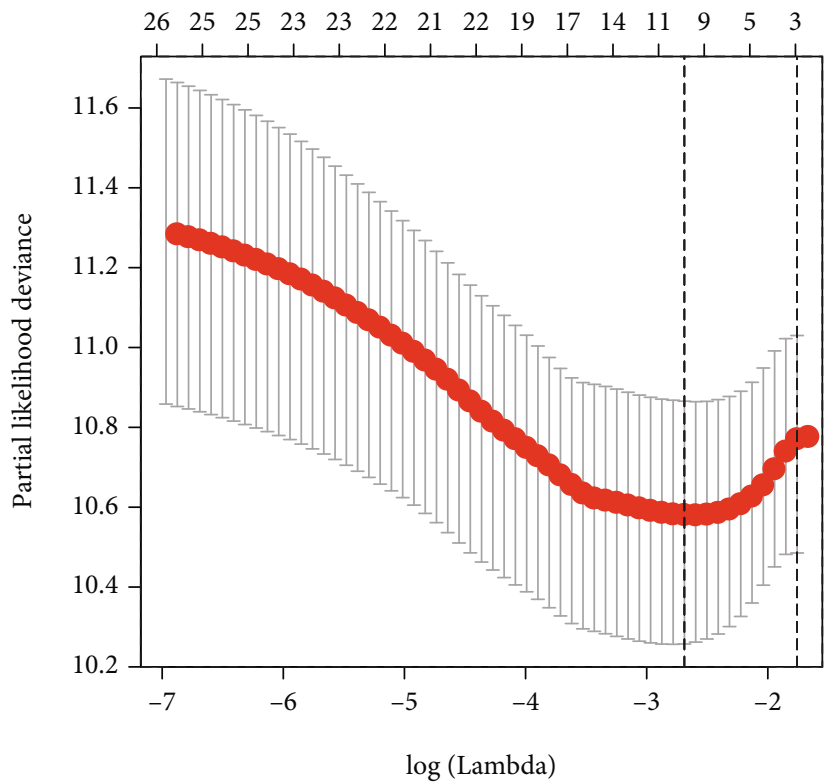

(c)

FIGURE 3: Univariate and LASSO regression analysis. (a) Univariate regression analysis of autophagy-related genes that can significantly affect OS in the TCGA training group. Notes: OS: overall survival; HCC: hepatocellular carcinoma. (b, c) LASSO regression analysis of autophagy-related genes that can significantly affect OS in the TCGA training group. Notes: LASSO: least absolute shrinkage and selection operator; OS: overall survival; TCGA: The Cancer Genome Atlas.

database for preserving genes associated with human autophagy. We acquired the gene symbols of 232 autophagy-related genes from the database. Then, we extracted the expression matrix of autophagy-related genes from 374 hepatocellular carcinoma patients in the TCGA database (https://portal.gdc. cancer.gov/) and obtained the clinicopathological features 
and prognostic information of HCC patients from the cBioPortal online website (https://www.cbioportal.org/). Differentially expressed ARGs between tumor samples and normal samples were determined by the Wilcoxon test. The criteria for screening differential genes were $\left|\log _{2} \mathrm{FC}\right|$ $>1$ and FDR $<0.05$. Normalization was performed by converting the expression matrix of the autophagy-related genes using the formula $\log _{2}(x+1)$. The staging of HCC patients was determined by using the 7th edition of AJCC (American Joint Committee on Cancer) staging.

2.2. Functional Enrichment Analysis of ARGs. Gene ontology (GO) is a database for the definition and description of genes and protein functions for a variety of species. GO annotations include molecular function (MF), biological process (BP), and cellular components (CC). Through these three functional categories, the function of a gene can be deeply described. Kyoto Encyclopedia of Genes and Genomes (KEGG) is a comprehensive database that integrates information on genomic, chemical, and system functions. Based on this database, we can further speculate on ARG-related signaling pathways. Then, we obtained the enrichment function and pathways of differentially expressed ARGs through the cluster profile package in $\mathrm{R}$ software.

2.3. Establishment and Validation of Autophagy-Related Gene Signature for OS. We matched the prognostic information of HCC patients with the liver cancer samples and finally obtained 367 HCC patients with prognostic information. A good predictive model should have internal and external validation, so we randomly divided the TCGA cohort into TCGA training $(n=183)$ and TCGA testing groups ( $n=184$ ) using the "sample" function in R language. Then, univariate regression analysis was used to explore differentially expressed ARGs that were significantly associated with the OS in the TCGA training group. The LASSO regression model was used to reduce the false-positive result caused by model overfitting. Furthermore, we constructed the OS gene signature by incorporating variables from multivariate regression analysis using the stepwise method. Finally, we validated the prognostic role of risk scores in the TCGA testing group, and three external datasets.

2.4. ARG Risk Score Computation. The ARG risk score of each HCC patient was computed by the expression value of the risk gene screened by multivariate regression analysis and the corresponding regression coefficient. The risk score for HCC patients is calculated as follows: risk score $=0.556$ * ATG10 + $0.217 *$ BIRC5 + $0.403 *$ GAPDH + $0.765 *$ TMEM74 for OS. Relative operating characteristic (ROC) analysis is used to determine the optimal cutoff value in each HCC dataset. Based on the optimal risk score of each dataset, we divided HCC patients into high-risk and low-risk groups. The Kaplan-Meier curve was used to map the survival time of patients in high- and low-risk groups, and the log-rank test was conducted to compare the significance of high- and low-risk groups.

2.5. RNA Extraction and $q R T-P C R$. We retrospectively analyzed the prognosis of $60 \mathrm{HCC}$ patients who underwent liver
TABLE 2: Multivariate Cox regression analysis of autophagy-related genes that can significantly affect OS in the TCGA training group.

\begin{tabular}{lccccc}
\hline Id & Coef & HR & HR.95L & HR.95H & $P$ value \\
\hline ATG10 & 0.556 & 1.744 & 0.913 & 3.332 & 0.092 \\
BIRC5 & 0.217 & 1.242 & 1.019 & 1.514 & 0.032 \\
GAPDH & 0.403 & 1.496 & 1.096 & 2.040 & 0.011 \\
TMEM74 & 0.765 & 2.150 & 1.378 & 3.353 & 0.001 \\
\hline
\end{tabular}

Notes: HR: hazards ratio; OS: overall survival; HCC: hepatocellular carcinoma.

resection at the First Affiliated Hospital of Anhui Medical University (AHMU) from 2010 to 2013. This study was authorized by the Ethics Committee of the First Affiliated Hospital of Anhui Medical University, and all patients signed informed consent. All HCC tumor tissues are stored in the refrigerator at -80 degrees. According to the instructions of the reagents, we used TRIzol (Invitrogen) to extract total RNA from tumor tissues and used the total RNA for reverse transcription to obtain cDNA (Takara Bio Inc.). Finally, we used SYBR Green reagent (Thermo Fisher) for real-time quantitative PCR. For qRT-PCR, each sample was repeated in triplicate. U6 serves as a reference standard for the quantification of each gene. The relative expression level of each gene was calculated using $2-\Delta \Delta \mathrm{CT}$. The qRTPCR was carried out with the Bio-Rad PCR thermocycling Instrument. The primer sequences of these genes are shown in Supplementary Table 2.

2.6. Statistical Analysis. Statistical analyses were analyzed using SPSS 24.0 (Chicago, IL, USA) and R 3.5.1 software (https://www.r-project.org/). We used R packages, including limma, pheatmap, ggpubr, clusterProfiler, survival, survminer, and survivalROC. The categorical variable is represented by frequency; if the continuous variable satisfies the normal distribution, it is expressed by mean \pm standard deviation; if it does not meet the normal distribution, it is expressed by median (interquartile range). Comparisons between categorical variables were utilized using chi-square analysis, and comparisons between continuous variables were performed using the Kruskal-Wallis test. Autophagyrelated risk genes were identified using the least absolute shrinkage and selection operator (LSAAO) and Cox regression model. Time-dependent ROC curves were used to analyze the performance of autophagy-related gene signature to predict overall survival. A $P$ value of below 0.05 was considered statistically significant.

\section{Results}

3.1. Clinicopathological Parameters of the Included Cohort. We randomly divided the entire TCGA dataset into training and testing groups, of which 183 were in the training group, and 184 were in the testing group. The entire dataset had 248 men and 119 women. The patients of AJCC stage I, II, III, and IV were $171,85,83$, and 4 , respectively. There were no significant differences in age, gender, AJCC staging, differentiation, survival status, and survival time between the 

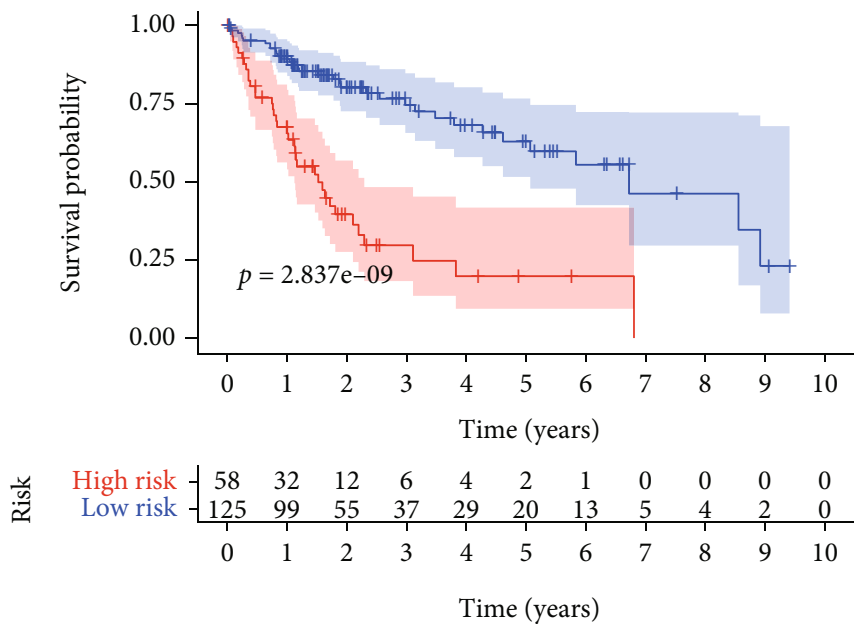

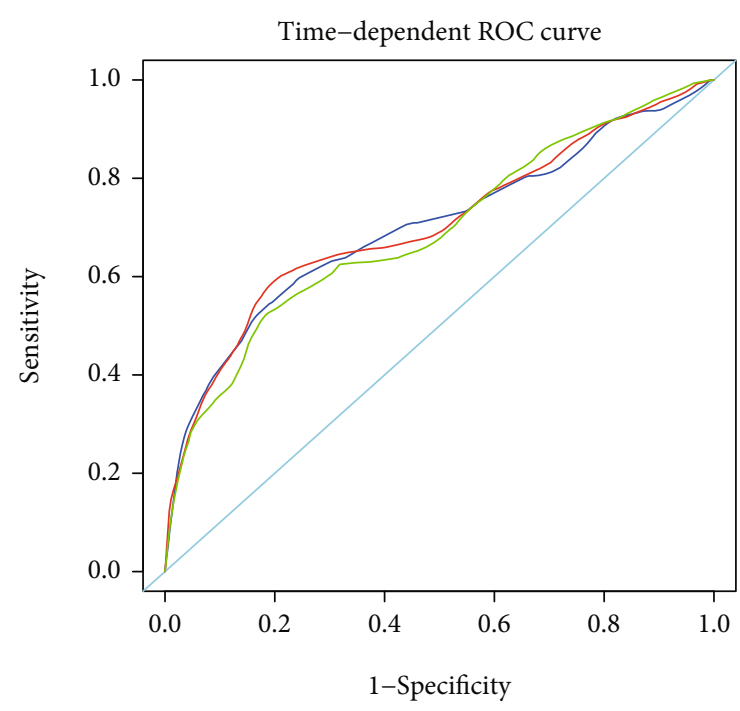

- AUC at 1 years: 0.701 AUC at 3 years: 0.704

- AUC at 5 years: 0.691

Risk
+ High risk
+ Low risk

(a)

(b)

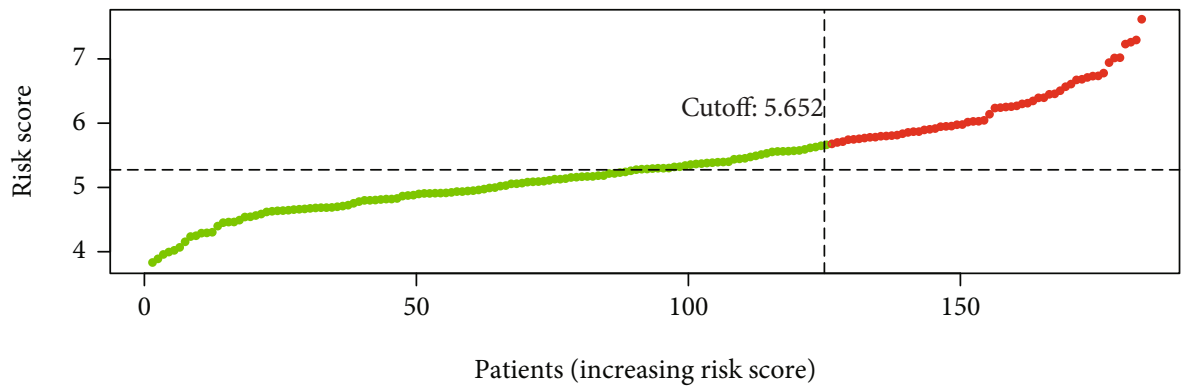

High risk

Low Risk

(c)

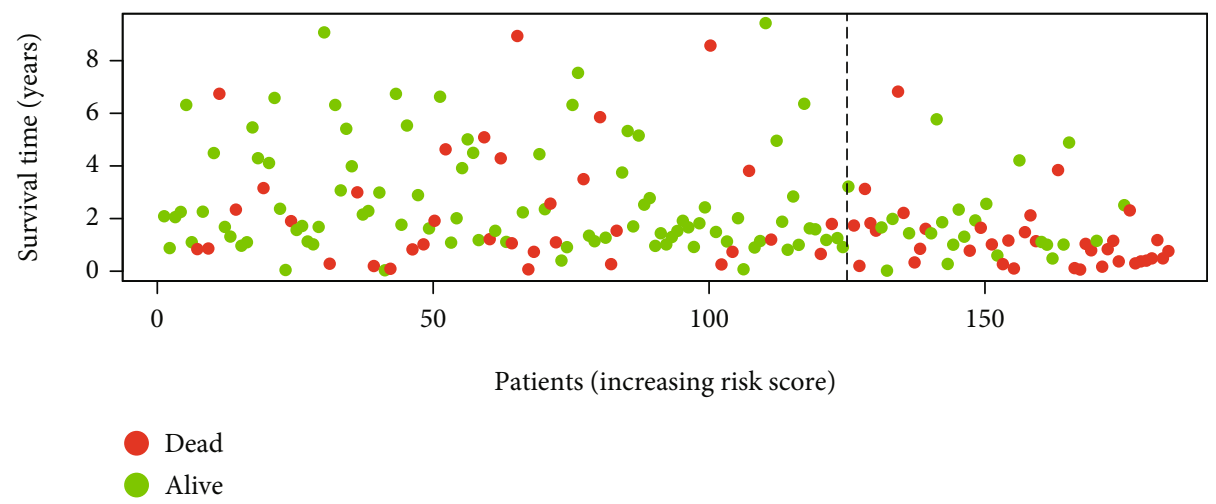

(d)

Figure 4: Continued. 


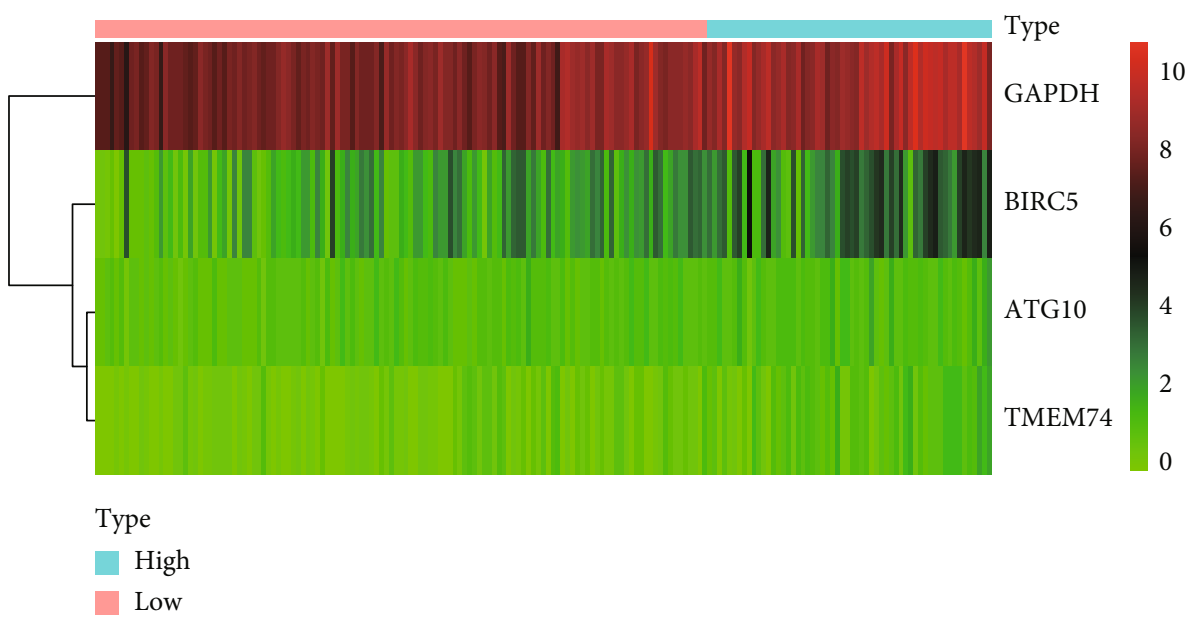

(e)

FIGURE 4: Autophagy-related gene signature for OS in the TCGA training group. (a) Prognostic value of the autophagy-related gene signature for OS in the TCGA training group; (b) diagnostic performance of the autophagy-related gene signature for OS in the TCGA training group; (c) the rank of the risk scores for HCC patients in the TCGA training group; (d) the survival status and time of HCC patients between the high- and low-risk groups for OS in the TCGA training group; (e) the heat map of the autophagy-related risk gene expression matrix for HCC patients in the high- and low-risk groups in the TCGA training group. Notes: OS: overall survival; HCC: hepatocellular carcinoma; TCGA: The Cancer Genome Atlas.

training group and the testing group (Table 1). Meanwhile, the clinicopathological characteristics of the ICGC, GSE116174, and the AHMU dataset are shown in Supplementary Table 1 .

\subsection{Identification of Differentially Expressed Autophagy-} Correlated Genes. We used the Wilcoxon test to analyze the expression matrix of tumor samples and normal samples. Based on the screening criteria $\left(\left|\log _{2} \mathrm{FC}\right|>1\right.$ and FDR $<0.05$ ), we obtained a total of 62 differentially expressed ARGs, of which 58 were upregulated, and 4 were downregulated. Volcano map of differentially expressed autophagyrelated genes is shown in Figure 1(a). Supplementary Figure 1 is a cluster heat map showing the expression matrix of differentially expressed autophagy-related genes, and Figure 1(b) is a box plot showing the expression levels of differentially expressed autophagy-related genes in tumor samples and normal samples. Among these differentially expressed ARGs, BIRC5 has the highest fold difference $\left(\log _{2} \mathrm{FC}=4.8\right)$, and its FDR value is $4.82 E-26$. At the same time, most of the differentially expressed ARGs are significantly increased in HCC tissues, thus revealing that they play an indispensable role in promoting the occurrence and development of liver cancer.

3.3. Functional Annotation of the Differentially Expressed Autophagy-Correlated Genes. Gene enrichment analysis can analyze the corresponding biological functions and pathways of selected genes. The biological process of GO is mainly enriched in autophagy, process utilizing autophagic mechanism, macroautophagy, regulation of autophagy, regulation of macroautophagy, regulation of apoptotic signaling pathway, autophagosome assembly, autophagosome organization, neuron death, and vacuumed organization (top 10).
The cellular components of GO are mainly enriched in autophagosome, vacuolar membrane, phagophore assembly site, autophagosome membrane, membrane region, late endosome, lysosomal membrane, lytic vacuole membrane, membrane raft, and membrane microdomain (top 10). The molecular function of GO is mainly enriched in protein kinase regulator activity, heat shock protein binding, cysteine-type endopeptidase activity, $\mathrm{BH}$ domain binding, kinase regulator activity, chaperone binding, calciumdependent cysteine-type endopeptidase activity, cysteinetype peptidase activity, ubiquitin-like protein ligase binding, and protein kinase activator activity (top 10) (Figure 2(a)). Similarly, the top 10 significantly enriched pathways are autophagy-animal, human papillomavirus infection, apoptosis, platinum drug resistance, longevity regulated pathway, autophagy-other, apoptosis-multiple species, measles, p53 signaling pathway, and cellular senescence (Figure 2(b)).

3.4. Identification of Autophagy-Related Risk Genes for Overall Survival. We performed univariate Cox regression analysis on 62 autophagy-related genes in the TCGA training group. The results of the univariate regression analysis showed that 26 autophagy-related genes were significantly associated with the prognosis of HCC patients $(P<0.05)$ (Figure 3(a)). In order to eliminate the false-positive results, we used the LASSO regression model to screen the independent variables further. The results of the LASSO regression analysis showed that 11 genes were significantly correlated with OS (Figures 3(b) and 3(c)). Finally, we put the selected 11 genes into multivariate regression analysis using the backward and forward method. The results of multivariate regression analysis identified ATG10, BIRC5, GAPDH, and TMEM74 are risk factors for OS in the TCGA training group (Table 2). 

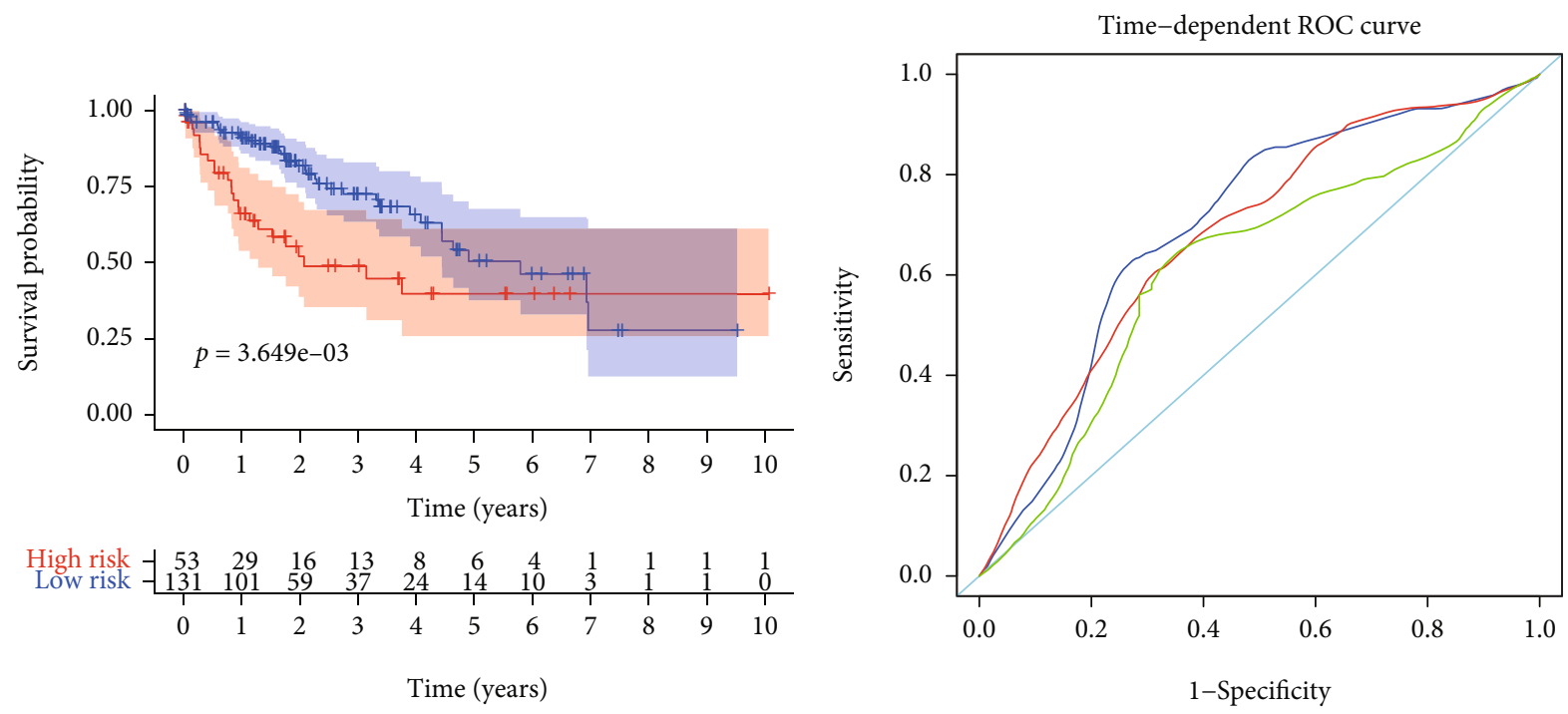

Risk
+ High risk
+ Low risk

— AUC at 1 years: 0.696

— AUC at 3 years: 0.681

— AUC at 5 years: 0.616

(a)

(b)

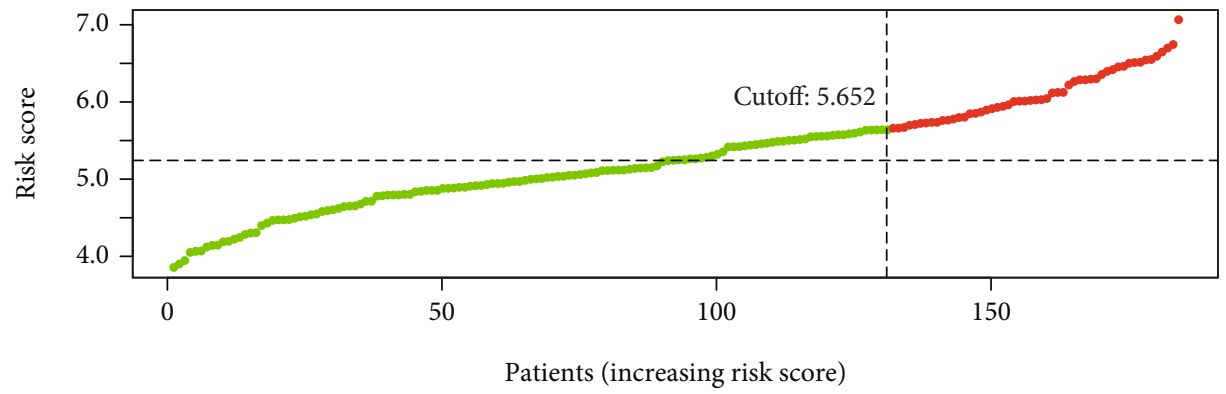

High risk

Low Risk

(c)

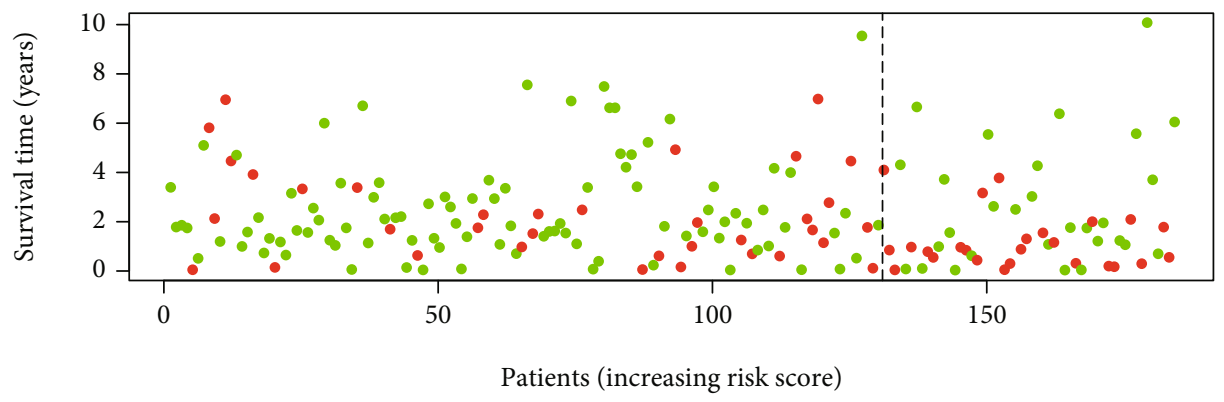

Dead

Alive

(d)

Figure 5: Continued. 


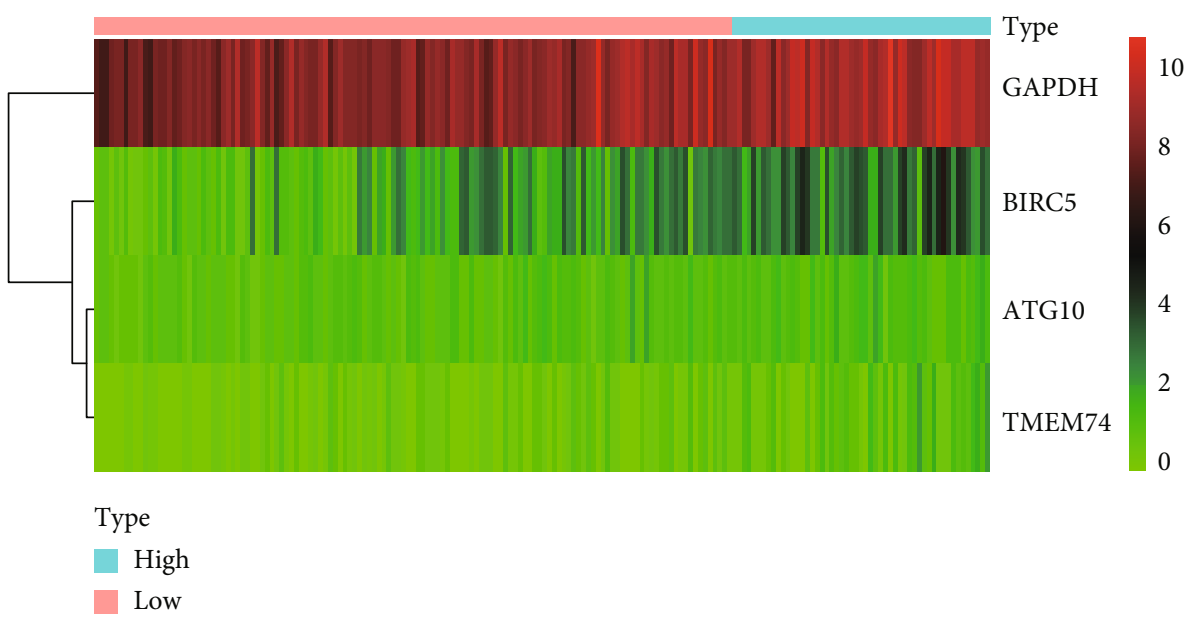

(e)

FIGURE 5: Autophagy-related gene signature for OS in the TCGA testing group. (a) Prognostic value of the autophagy-related gene signature for OS in the TCGA testing group; (b) diagnostic performance of the autophagy-related gene signature for OS in the TCGA testing group; (c) the rank of the risk scores for HCC patients in the TCGA testing group; (d) the survival status and time of HCC patients between the high- and low-risk groups for OS in the TCGA testing group; (e) the heat map of the autophagy-related risk gene expression matrix for HCC patients in the high- and low-risk groups in the TCGA testing group. Notes: OS: overall survival; HCC: hepatocellular carcinoma; TCGA: The Cancer Genome Atlas.

3.5. Development of Autophagy-Related Risk Gene Signature for Overall Survival. Based on the previous results of the multivariate regression analysis, we constructed a risk score formula for ARGs, which was calculated as follows: risk score $=0.556 *$ ATG $10+0.217 *$ BIRC5 $+0.403 * \mathrm{GAPDH}+$ $0.765 *$ TMEM74 for OS. According to the regression coefficient, it is evident that ATG10, BIRC5, GAPDH, and TMEM74 are all risk factors for HCC patients. According to the optimal cutoff value (5.652) of a risk score for the autophagy-related gene signature, we divided the TCGA HCC training cohort into high-risk and low-risk groups. The 1-year OS, 3-year OS, and 5-year OS rates of the highrisk group were remarkably lower than the 1-year OS, 3year OS, and 5-year OS of the low-risk group $(63.4 \%$ vs. $90.1 \% ; 29.7 \%$ vs. $74.5 \% ; 19.8 \%$ vs. $62.8 \%)(P=2.837 e-09)$ (Figure 4(a)). The 1-year AUC, 3-year AUC, and 5-year AUC of the autophagy-related gene signature for OS are $0.701,0.704$, and 0.691 in the TCGA training group (Figure 4(b)). Furthermore, we ranked the prognostic risk scores for OS in the TCGA training group. (Figure 4(c)). Figure $4(\mathrm{~d})$ shows the survival status and time of HCC patients for OS in the TCGA training group. Figure 4(e) shows the heat map of the risk ARG expression of HCC patients in the TCGA training group.

3.6. Validation of Autophagy-Related Risk Gene Signature for Overall Survival. The same cutoff value (5.652) of risk score in the TCGA training group was applied to the TCGA testing group. In the TCGA testing group, the 1-year OS, 3-year OS, and 5-year OS rates of the high-risk group were remarkably lower than the 1-year OS, 3-year OS, and 5-year OS of the low-risk group (66.1\% vs. $90.9 \% ; 48.8 \%$ vs. $72.5 \% ; 39.7 \%$ vs. 50.4\%) $(P=3.649 e-3)$ (Figure 5(a)). The 1-year AUC, 3 -year AUC, and 5-year AUC of the autophagy-related gene signature for OS are $0.696,0.681$, and 0.616 (Figure 5(b)). The risk score, survival status, and survival time of HCC patients in the TCGA testing group are plotted in Figures 5(c) and 5(d). Figure 5(e) shows the heat map of the ARG expression matrix of the HCC patients in the TCGA testing group. To further validate the role of our established formula, we explored the prognostic significance of autophagy-related gene signature in the external ICGC dataset, GSE116174 dataset, and AHMU dataset. In the ICGC dataset, the overall survival rate of the high-risk group was remarkably lower than that of the low-risk group $(P=9.45 e-8)$ (Figure 6(a)). The 1-year AUC, 3-year AUC, and 5-year AUC of the autophagy-related gene signature for OS are 0.685, 0.773, and 0.643 (Figure 6(b)). The risk score, survival status, survival time of HCC patients in the ICGC dataset are plotted in Figures 6(c) and 6(d). Figure 6(e) shows the heat map of the ARG expression matrix of the HCC patients in the ICGC dataset. The same tendency can be found in the GSE116174 dataset. The overall survival rate of the high-risk group was remarkably lower than that of the low-risk group $(P=7.67 e-4)$ (Figure $7(\mathrm{a}))$. The 1-year AUC, 3-year AUC, and 5-year AUC of the autophagy-related gene signature for OS is $0.685,0.646$, and 0.687 (Figure 7(b)). The risk score, survival status, and survival time of HCC patients in the GSE116174 dataset are plotted in Figures 7 (c) and 7(d). Figure 7(e) shows the heat map of the ARG expression matrix of the HCC patients in the GSE116174 dataset. Finally, we used our own dataset for verification. In the AHMU dataset, when compared with the low-risk group, patients in the high-risk group have a poorer prognosis $(P=3.43 e-3)$ (Figure $8(\mathrm{a}))$, and the autophagy-related gene signature shows a better predictive value for HCC (Figure 8(b)). The risk score, survival status, and survival time of HCC patients in the AHMU dataset 

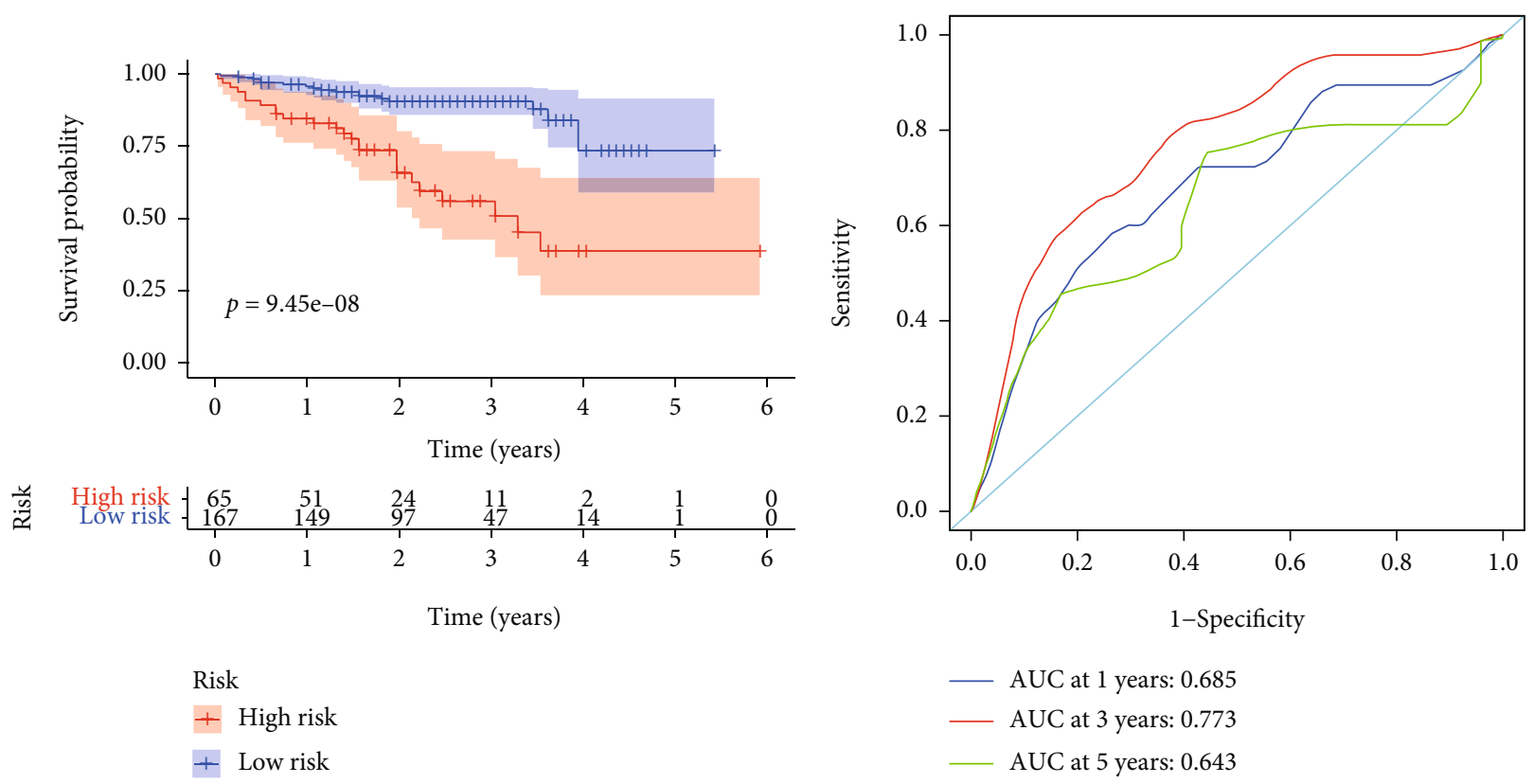

(a)

(b)

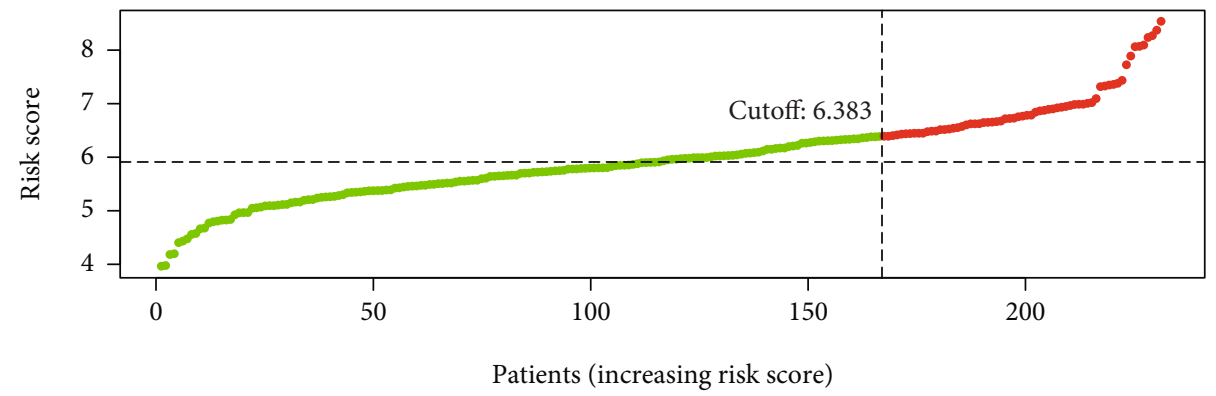

High risk

Low Risk

(c)

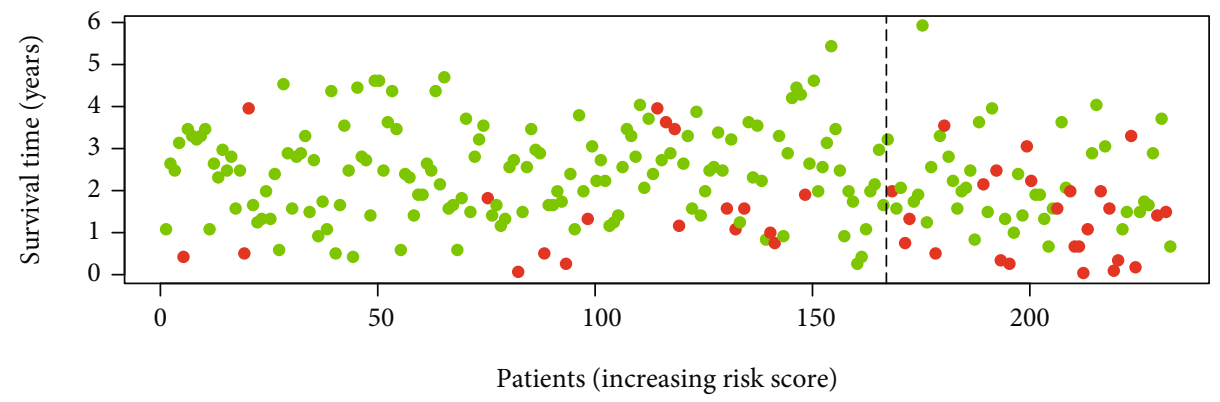

Dead

Alive

(d)

FIgUre 6: Continued. 


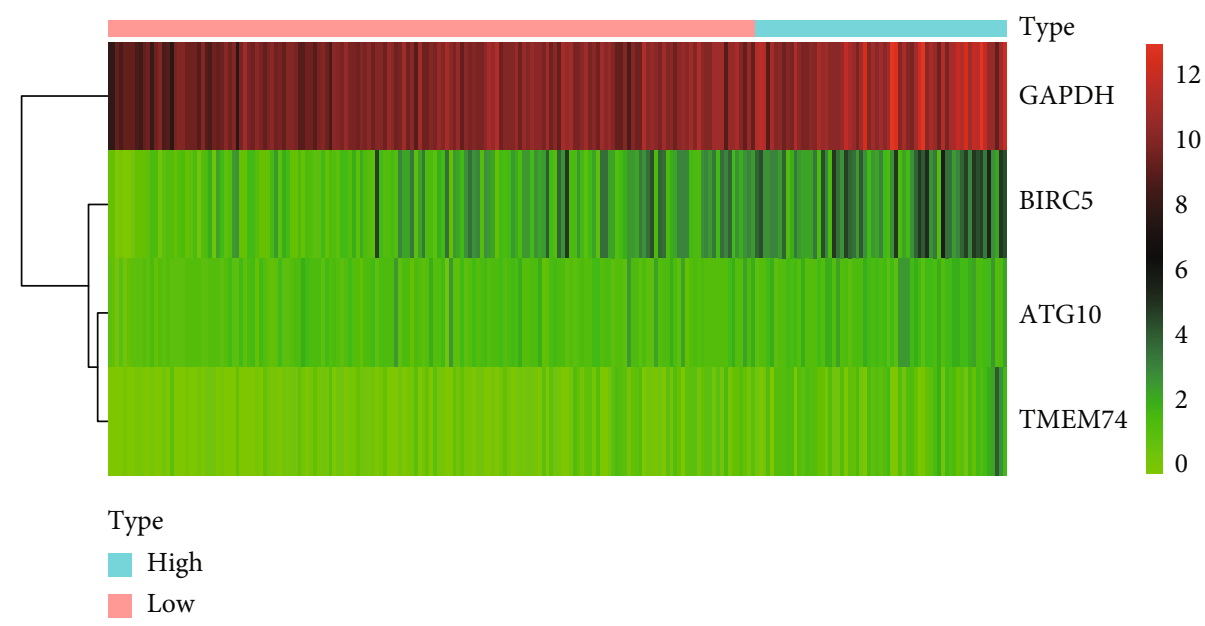

(e)

FIgURE 6: Autophagy-related gene signature for OS in the ICGC HCC dataset. (a) Prognostic value of the autophagy-related gene signature for OS in the ICGC HCC dataset; (b) diagnostic performance of the autophagy-related gene signature for OS in the ICGC HCC dataset; (c) the rank of the risk scores for HCC patients in the ICGC HCC dataset; (d) the survival status and time of HCC patients between the highand low-risk groups for OS in the ICGC HCC dataset; (e) the heat map of the autophagy-related risk gene expression matrix for HCC patients in the high- and low-risk groups in the ICGC HCC dataset. Notes: OS: overall survival; HCC: hepatocellular carcinoma; ICGC: International Cancer Genome Consortium.

are plotted in Figures 8(c) and 8(d). Figure 8(e) shows the heat map of the ARG expression matrix of the HCC patients in the AHMU dataset.

\subsection{Autophagy-Correlated Gene Signature Is Independently} Correlated with Overall Survival. To explore whether the ARG risk score is an independent risk factor for the prognosis of HCC patients in the TCGA training group, we included age, gender, AJCC stage, pathological grade, and the ARG risk score into the univariate regression model. Univariate regression analysis showed that the AJCC stage $(P=0.007)$ and ARG risk score $(P<0.001)$ were significantly associated with OS. Then, we included these factors into multivariate regression analysis. The results of the multivariate regression analysis showed that the AJCC stage $(P=0.042)$ and ARG risk score $(P<0.001)$ were significantly associated with OS (Table 3 ). Similarly, the AJCC stage and autophagy risk score were independent risk factors for the prognosis of HCC patients in the TCGA testing dataset (Table 4). Besides, in the external validation dataset, the autophagy risk score was also an independent risk factor for the prognosis of HCC patients in the ICGC dataset, GSE116174 dataset, and AHMU dataset (Tables 5-7). Therefore, the ARG risk score is an independent prognostic factor for OS in HCC patients.

3.8. Comparison of the Gene Signature We Constructed with the Published Gene Signatures. We also used the TCGA, ICGC, and GSE116174 datasets to analyze the predictive value of gene signature in the two published literature [12, 13 ] and the gene signature we constructed (Supplementary Figure 2). In the TCGA dataset, the results of the timedependent ROC curve show that the AUC of 8-gene signature (zhu-signature) [12] for 1-year and 5-year AUC is slightly higher than that of 4-gene signature (kongsignature) and 3-gene signature (lin-signature) [13], but its 3-year AUC is slighter lower than kong-signature and linsignature. The predicted value of 1-year, 3-year, and 5-year AUC is similar between kong-signature and lin-signature. In the ICGC dataset, the 1-year AUC of kong-signature is slightly lower than zhu-signature but was higher than linsignature and zhu-signature in both 3-year and 5-year AUC. In the GSE116174 dataset, kong-signature has shown good predictive value in 1-year, 3-year, and 5-year AUC. In summary, the kong-signature we constructed has a more accurate predictive value than the two other gene signatures in the previously published literature.

\section{Discussion}

With the development of science and technology in recent years, autophagy has gradually attracted the attention of researchers as an essential molecular process. Although many reports have explored the role of individual autophagy-related genes in liver cancer, research on the prognostic role of all autophagy-related genes in HCC is rarely investigated. Therefore, we deeply explored autophagy-related risk genes that affect the prognosis of HCC patients by digging into multiple public databases, so as to provide guidance for clinical evaluation of patients with hepatocellular carcinoma.

In our study, we downloaded and analyzed the RNA-seq data of the HCC cohort from TCGA and obtained 62 differentially expressed autophagy-related genes using the Wilcoxon test. Then, we performed gene enrichment analysis, and the function and pathway of autophagy-related genes play an essential role in the progression of hepatocellular carcinoma. Furthermore, we used univariate regression 

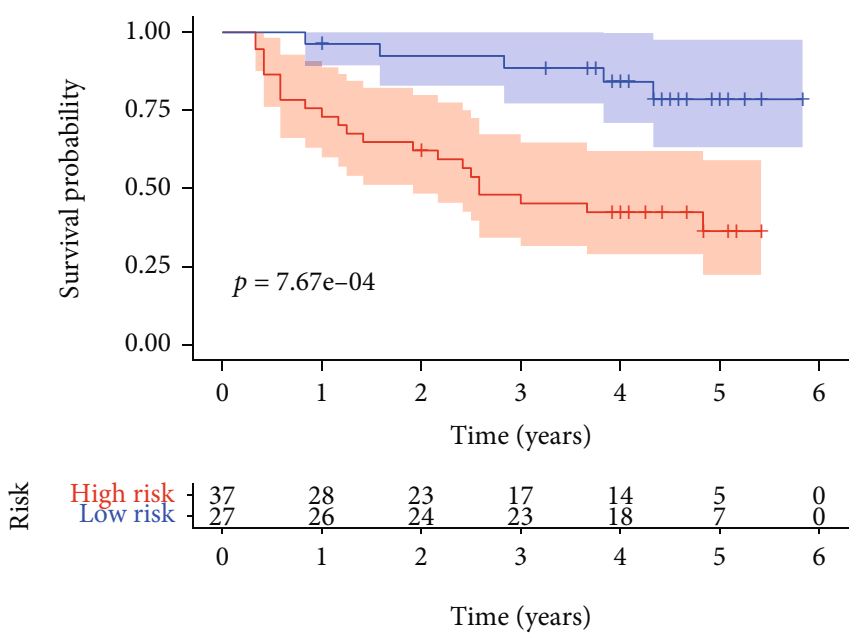

Risk

+ High risk

+ Low risk

(a)

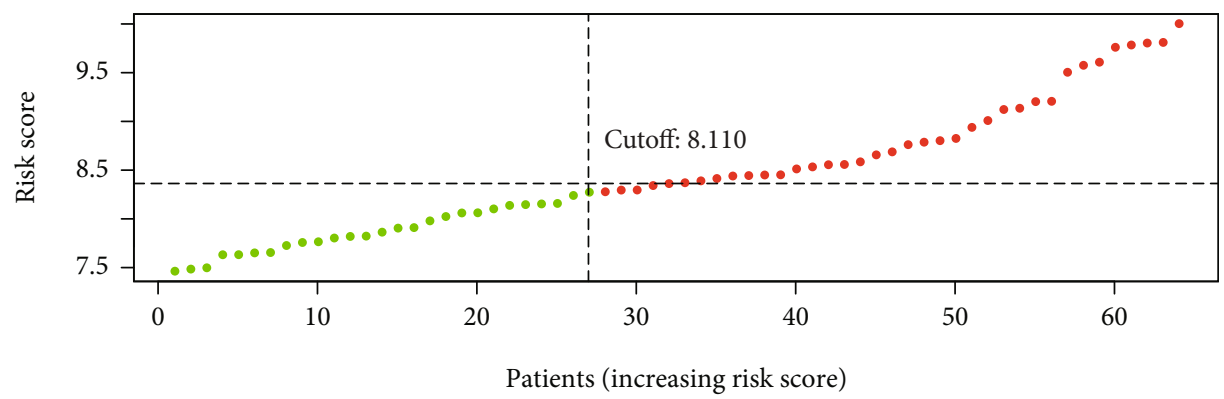

High risk

Low Risk

(c)

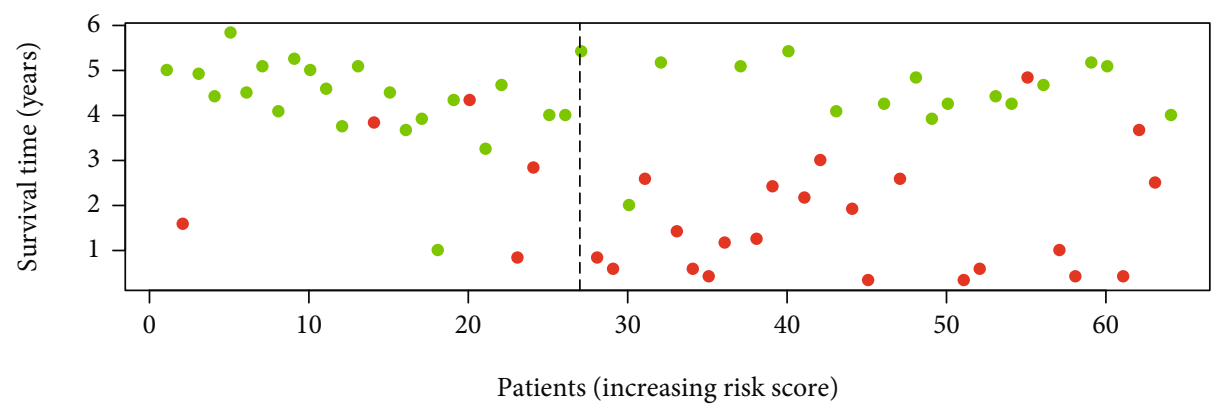

Dead

Alive

(d)

Figure 7: Continued.

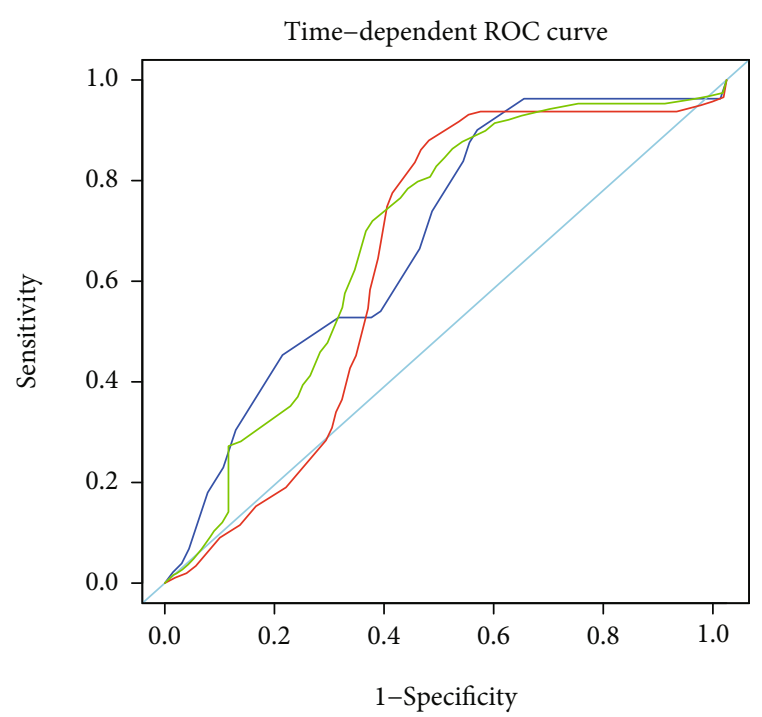

_ AUC at 1 years: 0.685

AUC at 3 years: 0.646

AUC at 5 years: 0.687 


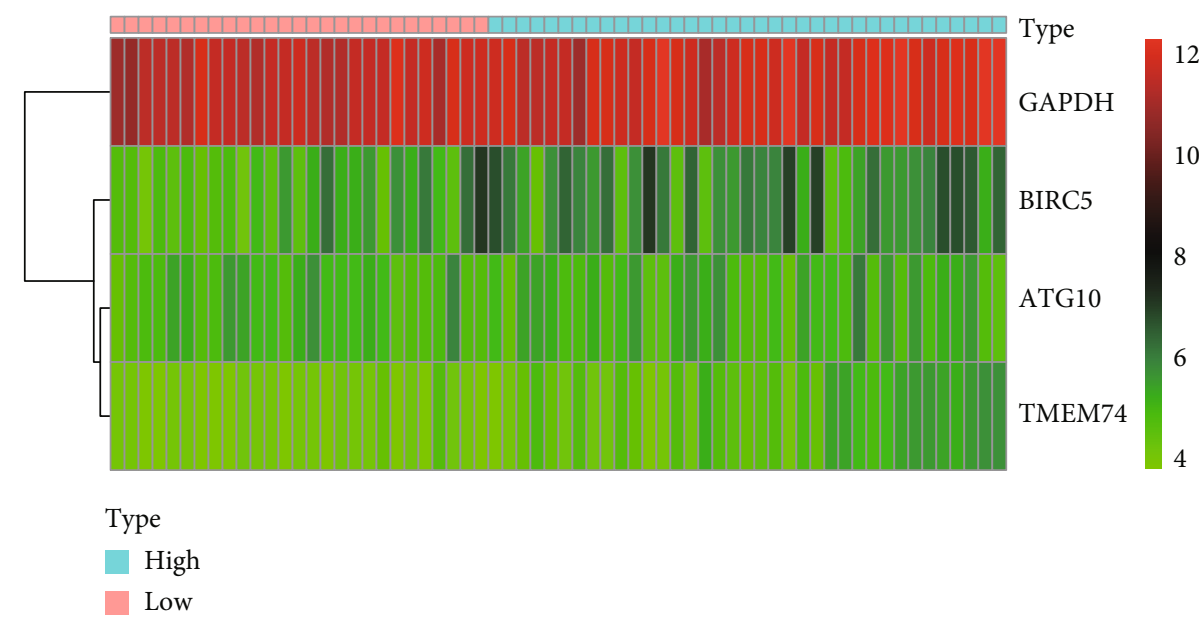

(e)

FIgURE 7: Autophagy-related gene signature for OS in the GSE116174 HCC dataset. (a) Prognostic value of the autophagy-related gene signature for OS in the GSE116174 HCC dataset; (b) diagnostic performance of the autophagy-related gene signature for OS in the GSE116174 HCC dataset; (c) the rank of the risk scores for HCC patients in the GSE116174 HCC dataset; (d) the survival status and time of HCC patients between the high- and low-risk groups for OS in the GSE116174 HCC dataset; (e) the heat map of the autophagyrelated risk gene expression matrix for HCC patients in the high- and low-risk groups in the GSE116174 HCC dataset. Notes: OS: overall survival; HCC: hepatocellular carcinoma.

analysis to explore the relationship between the expression of autophagy-related genes and the overall survival of HCC patients. The results showed that 26 autophagy-related genes were significantly related to OS. The LASSO regression and multivariate regression analysis were utilized for further screening, and we found that ATG10, BIRC5, GAPDH, and TMEM74 are risk ARGs for OS. Therefore, we established a prognostic gene signature for OS in the TCGA training group. The prognostic role of autophagy-related gene signature was also validated in the TCGA testing group, ICGC dataset, GSE116174 dataset, and AHMU dataset. Finally, we compared the gene signature we built with the published gene signatures. In our study, based on different statistical methods, we constructed a new 4-gene signature for the HCC prediction model. In both the internal training dataset and the external validation dataset, the 4-gene signature has better robustness and accuracy compared to the other two predictive signatures, which provides a new strategy for predicting the prognosis of liver cancer patients.

ATG10, also named ATG10L, encodes a protein that is involved in Ub-like modification, which is crucial for the formation of autophagosomes. ATG10 has been explored in a variety of tumors, including colorectal cancer, gastric cancer, non-small-cell lung cancer, and breast cancer [14-17]. For example, Jo et al.'s study found that increased expression of ATG10 is associated with vascular invasion, lymphatic metastasis, and poor prognosis in colorectal cancer [15]. Xie et al.'s research found that ATG10 can promote the proliferation and migration of lung cancer [17]. This is consistent with our study's finding that elevated expression of ATG10 is consistent with poor prognosis in patients with HCC.

BIRC5, also known as survivin, is a critical member of the apoptosis-inhibiting gene family, and it can encode reg- ulatory molecules that inhibit the death of apoptosis cells. The abnormal expression of BIRC5 is related to the malignant transformation of the cancer cell [18-23]. For example, the high expression of BIRC5 can promote the proliferation and angiogenesis of liver cancer cells, reduce the sensitivity to chemotherapy and radiotherapy, and suppress the apoptosis of tumor cells, thereby affecting the survival outcome of HCC patients [18]. Our study found that the high expression of BIRC5 is associated with poor prognosis in patients with HCC, which is also consistent with the published literature.

GAPDH is a well-known housekeeping gene. It includes a C-terminal catalytic domain and an N-terminal NAD + binding domain and plays an important role in the body's energy metabolism, DNA repair, autophagy, and cell proliferation [24-26]. Liu et al.'s research finds that GAPDH can convert glycolytic flux to serine metabolism by increasing PHGDH and promoting histone methylation, thereby promoting the progress of liver tumors in mice [26]. Ganapathy-Kanniappan et al.'s research found that injection of GAPDH antagonists into mouse liver cancer models can induce apoptosis of liver cancer cells and block the progression of Hep3B tumors, which may be used as a potential target therapy for HCC [25]. In our study, GAPDH was significantly upregulated in liver cancer, and high expression of GAPDH was an independent risk factor for prognosis in patients with liver cancer, which is consistent with the literature.

TMEM74, also known as FLJ30668 or NET36, is an autophagosome protein and lysosomal. It contains two TM domains and was first discovered by $\mathrm{Yu}$ et al. [27]. It plays a role in apoptosis, autophagy, tumor progression, and neurological diseases [28-32]. Sun et al.'s research finds that TMEM74 can induce autophagy through interaction with 

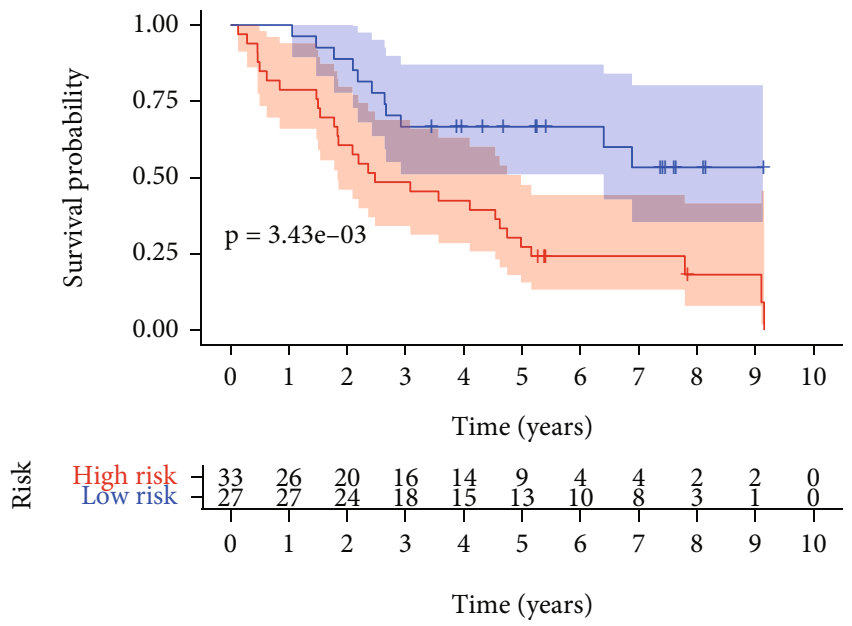

Risk

+ High risk

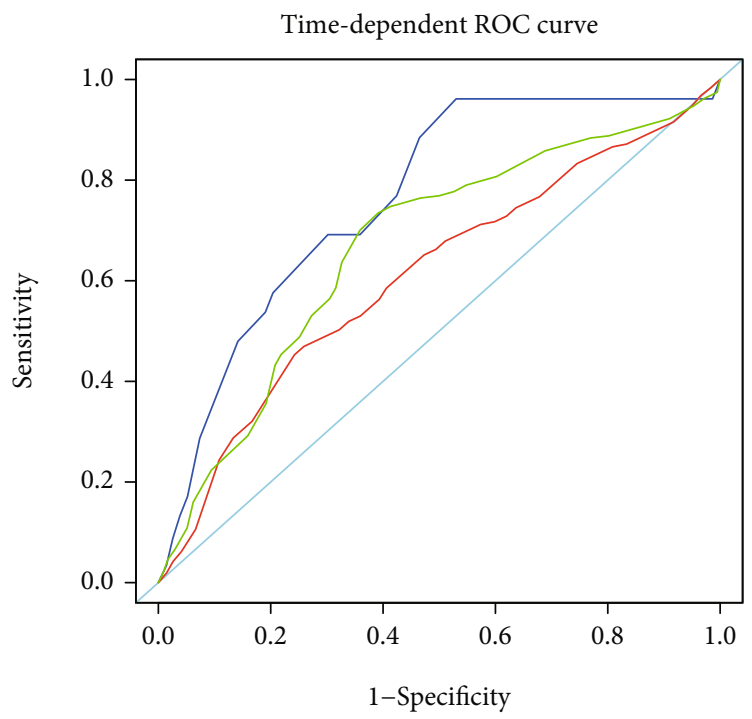

+ Low risk

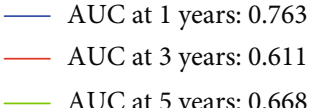

(a)

(b)

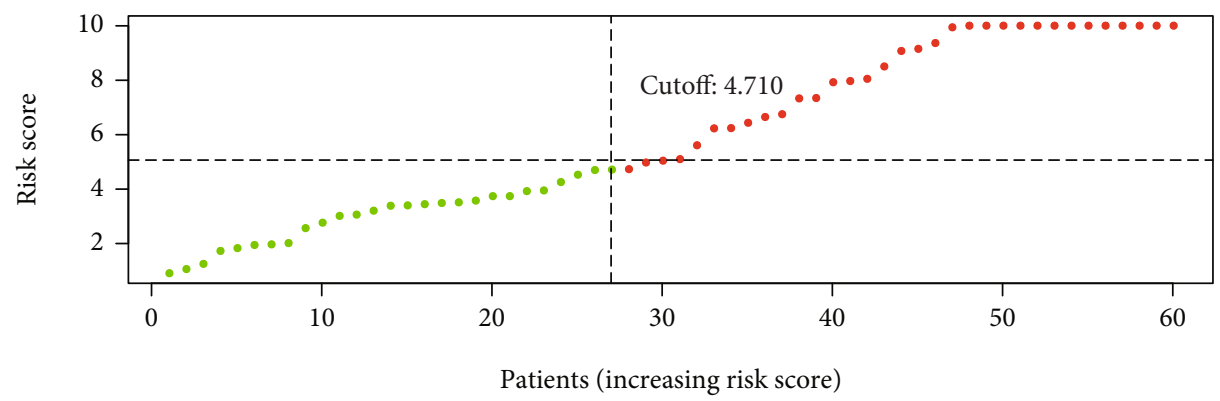

High risk

Low Risk

(c)

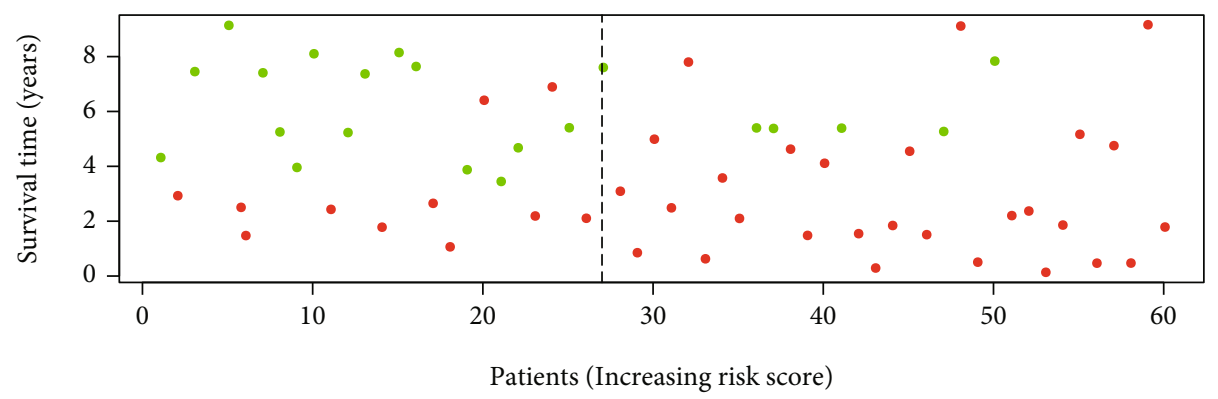

- Dead

- Alive

(d)

Figure 8: Continued. 


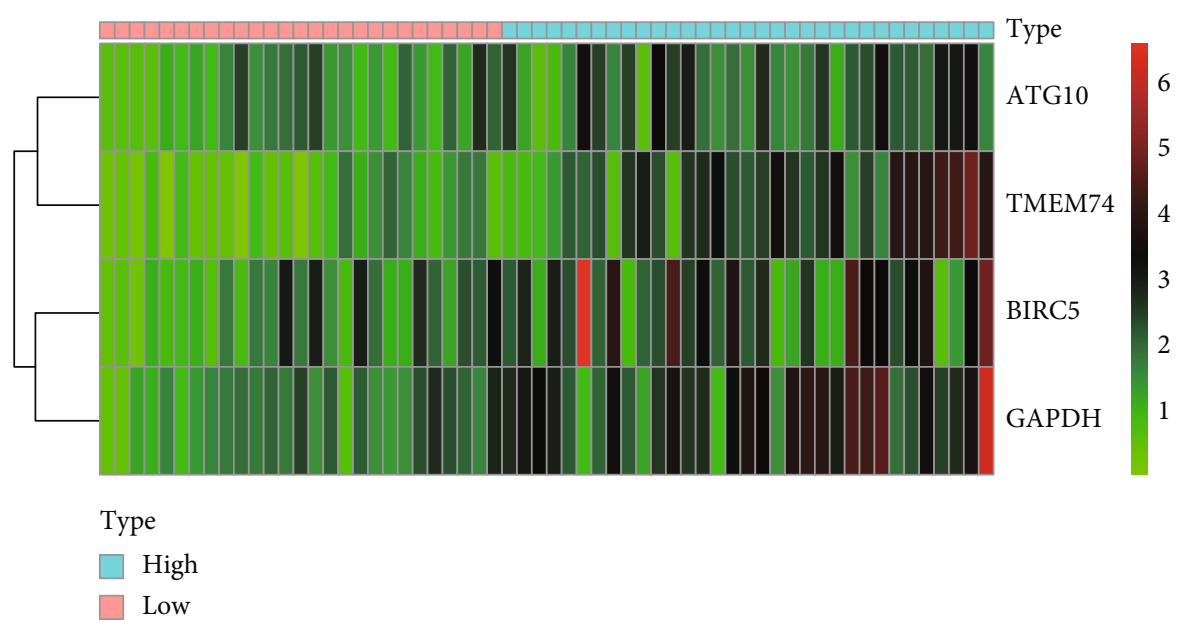

(e)

FIGURE 8: Autophagy-related gene signature for OS in the AHMU HCC dataset. (a) Prognostic value of the autophagy-related gene signature for OS in the AHMU HCC dataset; (b) diagnostic performance of the autophagy-related gene signature for OS in the AHMU HCC dataset; (c) the rank of the risk scores for HCC patients in the AHMU HCC dataset; (d) the survival status and time of HCC patients between the high- and low-risk groups for OS in the AHMU HCC dataset; (e) the heat map of the autophagy-related risk gene expression matrix for HCC patients in the high- and low-risk groups in the AHMU HCC dataset. Notes: OS: overall survival; AHMU: Anhui Medical University; HCC: hepatocellular carcinoma.

TABLE 3: Univariate and multivariate regression analyses of OS in HCC patients of the TCGA training group.

\begin{tabular}{lccccc}
\hline \multirow{2}{*}{ Variable } & \multicolumn{2}{c}{ Univariate analysis } & \multicolumn{2}{c}{$\begin{array}{c}\text { Multivariate analysis } \\
\text { Hazard ratio }\end{array}$ 95\% confidence interval } \\
\hline Age $(\geq 60 /<60)$ & 0.220 & 1.378 & $0.826-2.301$ & & \\
Sex (female/male) & 0.776 & 1.079 & $0.639-1.822$ & & 1.745 \\
AJCC stage (IV + III/II + I) & 0.007 & 2.061 & $1.216-3.494$ & 0.042 & $1.021-2.983$ \\
Histologic grade (G4 + G3/G2 + G1) & 0.276 & 0.744 & $0.437-1.266$ & & 3.775 \\
Risk score (high/low) & $<0.001$ & 4.027 & $2.410-6.728$ & $<0.001$ & $2.251-6.328$ \\
\hline
\end{tabular}

Notes: AJCC: American Joint Committee on Cancer; OS: overall survival; HCC: hepatocellular carcinoma; TCGA: The Cancer Genome Atlas.

TABLE 4: Univariate and multivariate regression analyses of OS in HCC patients of the TCGA testing group.

\begin{tabular}{|c|c|c|c|c|c|c|}
\hline \multirow{2}{*}{ Variable } & \multicolumn{3}{|c|}{ Univariate analysis } & \multicolumn{3}{|c|}{ Multivariate analysis } \\
\hline & $P$ & Hazard ratio & $95 \%$ confidence interval & $P$ & Hazard ratio & $95 \%$ confidence interval \\
\hline Age $(\geq 60 /<60)$ & 0.808 & 1.071 & $0.616-1.863$ & & & \\
\hline Sex (female/male) & 0.082 & 1.637 & $0.940-2.852$ & & & \\
\hline AJCC stage (IV + III/II + I) & $<0.001$ & 3.495 & $2.002-6.100$ & $<0.001$ & 3.545 & $2.017-6.231$ \\
\hline Histologic grade $(\mathrm{G} 4+\mathrm{G} 3 / \mathrm{G} 2+\mathrm{G} 1)$ & 0.025 & 1.886 & $1.084-3.280$ & 0.036 & 1.831 & $1.041-3.221$ \\
\hline Risk score (high/low) & 0.006 & 2.201 & $1.259-3.850$ & 0.020 & 1.947 & $1.109-3.416$ \\
\hline
\end{tabular}

Notes: AJCC: American Joint Committee on Cancer; OS: overall survival; HCC: hepatocellular carcinoma; TCGA: The Cancer Genome Atlas.

ATG16L1 and ATG9A, thereby promoting tumor cell survival [30]. Sun et al.'s study found that TMEM74 is highly expressed in liver cancer and lung cancer tissues and correlated to the poor prognosis of cancer patients. After overexpressing TMEM74 in tumor cells, the proliferation ability of tumor cells was enhanced [31]. In our study, elevated expression of TEME74 was associated with poor prognosis in patients with liver cancer, which is in accordance with the study by Sun et al.

However, there are still deficiencies in our research. Firstly, the data we use is derived from public databases, and these findings require subsequent verification. Secondly, we have discovered some potential biomarkers of liver cancer and have not further explored the underlying 
TABLE 5: Univariate and multivariate regression analyses of OS in HCC patients of the ICGC dataset.

\begin{tabular}{|c|c|c|c|c|c|c|}
\hline \multirow{2}{*}{ Variable } & \multicolumn{3}{|c|}{ Univariate analysis } & \multicolumn{3}{|c|}{ Multivariate analysis } \\
\hline & $P$ value & Hazard ratio & $95 \%$ confidence interval & $P$ value & Hazard ratio & 95\% confidence interval \\
\hline Age $(\geq 60 /<60)$ & 0.590 & 0.823 & $0.405-1.671$ & & & \\
\hline Sex (female/male) & 0.039 & 1.926 & $1.033-3.590$ & 0.017 & 2.231 & $1.152-4.322$ \\
\hline AJCC stage $(\mathrm{IV}+\mathrm{III} / \mathrm{II}+\mathrm{I})$ & 0.005 & 2.384 & $1.304-4.359$ & 0.002 & 2.726 & $1.437-5.173$ \\
\hline Prior malignancy (yes/no) & 0.180 & 1.750 & $0.773-3.963$ & & & \\
\hline Risk (high/low) & $<0.001$ & 4.528 & $2.462-8.328$ & $<0.001$ & 4.084 & $2.214-7.533$ \\
\hline
\end{tabular}

Notes: AJCC: American Joint Committee on Cancer; OS: overall survival; HCC: hepatocellular carcinoma; ICGC: International Cancer Genome Consortium.

TABLE 6: Univariate and multivariate regression analyses of OS in HCC patients of the GSE116174 dataset.

\begin{tabular}{|c|c|c|c|c|c|c|}
\hline \multirow{2}{*}{ Variable } & \multicolumn{3}{|c|}{ Univariate analysis } & \multicolumn{3}{|c|}{ Multivariate analysis } \\
\hline & $P$ value & Hazard ratio & 95\% confidence interval & $P$ value & Hazard ratio & 95\% confidence interval \\
\hline Age $(\geq 60 /<60)$ & 0.788 & 0.892 & $0.387-2.055$ & & & \\
\hline Sex (female/male) & 0.250 & 0.309 & $0.042-2.287$ & & & \\
\hline Alcohol (yes/no) & 0.968 & 0.980 & $0.369-2.603$ & & & \\
\hline Smoke (yes/no) & 0.339 & 1.459 & $0.673-3.163$ & & & \\
\hline AJCC stage $(\mathrm{IV}+\mathrm{III} / \mathrm{II}+\mathrm{I})$ & 0.300 & 1.622 & $0.651-4.043$ & & & \\
\hline Risk (high/low) & 0.003 & 4.429 & $1.664-11.788$ & 0.003 & 4.429 & $1.664-11.788$ \\
\hline
\end{tabular}

Notes: AJCC: American Joint Committee on Cancer; OS: overall survival; HCC: hepatocellular carcinoma.

TABLE 7: Univariate and multivariate regression analyses of OS in HCC patients of the AHMU dataset.

\begin{tabular}{|c|c|c|c|c|c|c|}
\hline \multirow{2}{*}{ Variable } & \multicolumn{3}{|c|}{ Univariate analysis } & \multicolumn{3}{|c|}{ Multivariate analysis } \\
\hline & $P$ value & Hazard ratio & 95\% confidence interval & $P$ value & Hazard ratio & 95\% confidence interval \\
\hline Age $(\geq 60 /<60)$ & 0.908 & 0.962 & $0.497-1.861$ & & & \\
\hline Sex (female/male) & 0.664 & 0.841 & $0.385-1.839$ & & & \\
\hline AJCC stage (IV + III/II + I) & 0.014 & 2.303 & $1.184-4.480$ & - & & \\
\hline Cirrhosis (yes/no) & 0.330 & 0.721 & $0.374-1.391$ & & & \\
\hline Tumor size $(>5 / \leq 5 \mathrm{~cm})$ & 0.432 & 1.296 & $0.679-2.473$ & & & \\
\hline Tumor number (multiple/single) & 0.004 & 2.687 & $1.365-5.291$ & 0.013 & 2.372 & $1.203-4.676$ \\
\hline Differentiation (poor/well + moderate) & 0.471 & 1.265 & $0.667-2.398$ & & & \\
\hline Risk (high/low) & 0.005 & 2.752 & $1.358-5.575$ & 0.011 & 2.517 & $1.238-5.119$ \\
\hline
\end{tabular}

Notes: AJCC: American Joint Committee on Cancer; OS: overall survival; HCC: hepatocellular carcinoma.

mechanism of its function, and subsequent research is needed to explore it.

\section{Conclusion}

In this research, we found autophagy-related risk scores can significantly distinguish high- and low-risk groups of HCC patients and are also significantly related to the prognosis of HCC patients. Therefore, the prediction model based on autophagy-related genes may be used to predict the prognosis of HCC patients to provide a new strategy for the prevention of HCC in the clinic.

\section{Data Availability}

We use public databases for subsequent analysis, and the corresponding data can be found in The Human Autophagy Database (HADb), The Cancer Genome Atlas (TCGA),
International Cancer Genome Consortium (ICGC), Gene Expression Omnibus (GEO), and the cBioPortal.

\section{Conflicts of Interest}

The authors stated that there are no conflicts of interest.

\section{Authors' Contributions}

Jianlin Zhang, Min Zhang, and Jin Huang contributed equally to this manuscript.

\section{Acknowledgments}

This research was funded by the 2020 Anhui Medical University School Fund (project: No. 2020xkj180), Wu Jieping Medical Foundation (project: 320.6750.2020-17-5), and the 
Youth Training Program of the First Affiliated Hospital of Anhui Medical University (project: 2021kj24).

\section{Supplementary Materials}

Supplementary Figure 1: cluster heat map of differentially expressed autophagy-related genes in HCC patients from the TCGA cohort. Supplementary Figure 2: comparison of the gene signature we constructed with the published gene signatures. Supplementary Table 1: clinicopathological characteristics of the ICGC, GSE116174, and the AHMU dataset. Supplementary Table 2: primers used for PCR. (Supplementary Materials)

\section{References}

[1] M. M. Gadsden and D. E. Kaplan, "Multidisciplinary approach to HCC management: how can this be done?," Digestive Diseases and Sciences, vol. 64, no. 4, pp. 968-975, 2019.

[2] R. L. Siegel, K. D. Miller, and A. Jemal, "Cancer statistics, 2018," CA: A Cancer Journal for Clinicians, vol. 68, no. 1, pp. 7-30, 2018.

[3] Y. Boyao, S. Mengjiao, B. Caicai, L. Xiaoling, L. Zhenxing, and W. Manxia, "Dynamic expression of autophagy-related factors in autoimmune encephalomyelitis and exploration of curcumin therapy," Journal of Neuroimmunology, vol. 337, p. $577067,2019$.

[4] C. G. Towers, D. Wodetzki, and A. Thorburn, "Autophagy and cancer: modulation of cell death pathways and cancer cell adaptations," The Journal of Cell Biology, vol. 219, p. jcb.201909033, 2019.

[5] J. L. Wang and C. J. Xu, "Astrocytes autophagy in aging and neurodegenerative disorders," Biomedicine \& Pharmacotherapy, vol. 122, p. 109691, 2020.

[6] X. Wen and D. J. Klionsky, "At a glance: a history of autophagy and cancer," The Journal of Cell Biology, vol. 66, 2019.

[7] J. Madrigal-Matute and A. M. Cuervo, "Regulation of liver metabolism by autophagy," Gastroenterology, vol. 150, no. 2, pp. 328-339, 2016.

[8] J. L. Schneider, J. Villarroya, A. Diaz-Carretero et al., "Loss of hepatic chaperone-mediated autophagy accelerates proteostasis failure in aging," Aging Cell, vol. 14, no. 2, pp. 249-264, 2015.

[9] M. Kon, R. Kiffin, H. Koga et al., "Chaperone-mediated autophagy is required for tumor growth," Science Translational Medicine, vol. 3, no. 109, pp. 109-117, 2011.

[10] Y. Inami, S. Waguri, A. Sakamoto et al., "Persistent activation of Nrf2 through p62 in hepatocellular carcinoma cells," The Journal of Cell Biology, vol. 193, no. 2, pp. 275-284, 2011.

[11] H. M. Ni, B. L. Woolbright, J. Williams et al., "Nrf2 promotes the development of fibrosis and tumorigenesis in mice with defective hepatic autophagy," Journal of Hepatology, vol. 61, no. 3, pp. 617-625, 2014.

[12] Y. Zhu, R. Wang, W. Chen, Q. Chen, and J. Zhou, "Construction of a prognosis-predicting model based on autophagyrelated genes for hepatocellular carcinoma (HCC) patients," Aging, vol. 12, no. 14, pp. 14582-14592, 2020.

[13] P. Lin, R. Q. He, Y. W. Dang et al., “An autophagy-related gene expression signature for survival prediction in multiple cohorts of hepatocellular carcinoma patients," Oncotarget, vol. 9, no. 25, pp. 17368-17395, 2018.
[14] Q. H. Cao, F. Liu, Z. L. Yang et al., "Prognostic value of autophagy related proteins ULK1, Beclin 1, ATG3, ATG5, ATG7, ATG9, ATG10, ATG12, LC3B and p62/SQSTM1 in gastric cancer," American Journal of Translational Research, vol. 8, no. 9, pp. 3831-3847, 2016.

[15] Y. K. Jo, S. C. Kim, I. J. Park et al., "Increased expression of ATG10 in colorectal cancer is associated with lymphovascular invasion and lymph node metastasis," PLoS One, vol. 7, no. 12, article e52705, 2012.

[16] Z. Qin, J. Xue, Y. He et al., "Potentially functional polymorphisms in _ATG10_are associated with risk of breast cancer in a Chinese population," Gene, vol. 527, no. 2, pp. 491-495, 2013.

[17] K. Xie, C. Liang, Q. Li et al., "Role ofATG10 expression quantitative trait loci in non-small cell lung cancer survival," International Journal of Cancer, vol. 139, no. 7, pp. 1564-1573, 2016.

[18] L. Cao, C. Li, S. Shen et al., "OCT4 increases BIRC5 and CCND1 expression and promotes cancer progression in hepatocellular carcinoma," BMC Cancer, vol. 13, no. 1, 2013.

[19] A. S. Hamy, I. Bieche, J. Lehmann-Che et al., "BIRC5 (survivin): a pejorative prognostic marker in stage II/III breast cancer with no response to neoadjuvant chemotherapy," Breast Cancer Research and Treatment, vol. 159, no. 3, pp. 499-511, 2016.

[20] S. H. Liu, Y. Hong, S. Markowiak et al., "BIRC5 is a target for molecular imaging and detection of human pancreatic cancer," Cancer Letters, vol. 457, pp. 10-19, 2019.

[21] X. Shang, G. Liu, Y. Zhang et al., "Downregulation of BIRC5 inhibits the migration and invasion of esophageal cancer cells by interacting with the PI3K/Akt signaling pathway," Journal of Oncology, vol. 16, no. 3, 2018.

[22] N. A. Vaishlia, M. V. Zinov'eva, A. V. Sass, E. P. Kopantsev, T. V. Vinogradova, and E. D. Sverdlov, "Increase of BIRC5 gene expression in non-small cell lung cancer and esophageal squamous cell carcinoma does not correlate with expression of genes SMAC/DIABLO and PML encoding its inhibitors," Molekuliarnaia Biologiia (Mosk), vol. 42, no. 4, pp. 652-661, 2008.

[23] C. Wang, X. Zheng, C. Shen, and Y. Shi, "MicroRNA-203 suppresses cell proliferation and migration by targeting BIRC5 and LASP1 in human triple-negative breast cancer cells," Journal of Experimental \& Clinical Cancer Research, vol. 31, p. 58, 2012.

[24] S. Ganapathy-Kanniappan, R. Kunjithapatham, and J. F. Geschwind, "Glyceraldehyde-3-phosphate dehydrogenase: a promising target for molecular therapy in hepatocellular carcinoma," Oncotarget, vol. 3, no. 9, pp. 940-953, 2012.

[25] S. Ganapathy-Kanniappan, R. Kunjithapatham, M. S. Torbenson et al., "Human hepatocellular carcinoma in a mouse model: assessment of tumor response to percutaneous ablation by using glyceraldehyde-3-phosphate dehydrogenase antagonists," Radiology, vol. 262, no. 3, pp. 834-845, 2012.

[26] S. Liu, Y. Sun, M. Jiang et al., "Glyceraldehyde-3-phosphate dehydrogenase promotes liver tumorigenesis by modulating phosphoglycerate dehydrogenase," Hepatology, vol. 66, no. 2, pp. 631-645, 2017.

[27] C. Yu, L. Wang, B. Lv et al., "TMEM74, a lysosome and autophagosome protein, regulates autophagy," Biochemical and Biophysical Research Communications, vol. 369, no. 2, pp. 622-629, 2008. 
[28] L. X. Shao, Q. Jiang, X. X. Liu et al., "Selective inhibition of Tmem74 expression in BLA pyramidal neurons," Molecular Psychiatry, vol. 24, no. 10, p. 1399, 2019.

[29] L. X. Shao, Q. Jiang, X. X. Liu et al., "Functional coupling of Tmem74 and HCN1 channels regulates anxiety-like behavior in BLA neurons," Molecular Psychiatry, vol. 24, no. 10, pp. 1461-1477, 2019.

[30] Y. Sun, Y. Chen, J. Zhang et al., “TMEM74 promotes tumor cell survival by inducing autophagy via interactions with ATG16L1 and ATG9A," Cell Death \& Disease, vol. 8, no. 8, article e3031, 2017.

[31] Y. Sun, J. Deng, P. Xia, W. Chen, and L. Wang, “The expression of TMEM74 in liver cancer and lung cancer correlating with survival outcomes," Applied Immunohistochemistry \& Molecular Morphology, vol. 27, no. 8, pp. 618-625, 2019.

[32] Y. Sun, Q. Li, J. Zhang et al., “Autophagy regulatory molecule, TMEM74, interacts with BIK and inhibits BIK- induced apoptosis," Cellular Signalling, vol. 36, pp. 34-41, 2017. 